\title{
Space-Time Quasicrystal Structures and Inflationary and Late Time Evolution Dynamics in Accelerating Cosmology Sergiu I. Vacaru*
}

\author{
Physics Department, California State University at Fresno, CA 93740, USA \\ and Project IDEI, University "Al. I. Cuza" Iaşi, Romania
}

October 2, 2018

\begin{abstract}
We construct new classes of cosmological solutions in modified and Einstein gravity theories encoding space-time quasicrystal, STQC, configurations modeled by nonlinear self-organized and pattern forming quasi-periodic structures. Such solutions are defined by generic off-diagonal locally anisotropic and inhomogeneous metrics depending via generating and integration functions on all spacetime coordinates. There are defined nonholonomic variables and conditions for the generating/integration functions and sources for effective descriptions, or approximations, as "quasi" Friedmann-Lamaître-Robertson-Walker (FLRW) metrics. Such (off-) diagonal STQCFLRW configurations contain memory on nonlinear classical and/or quantum interactions and may describe new acceleration cosmology scenarios. For special time-periodic conditions on nonlinear gravitational and matter field interactions, we can model at cosmological scales certain analogous of time crystal like structures originally postulated by Frank Wilczek in condensed matter physics. We speculate how STQC quasi-FLRW configurations could explain modern cosmology data and provide viable descriptions for the inflation and structure formation in our Universe. Finally, it is discussed systematically and critically how a unified description of inflation with dark energy era can be explained by (modified) cosmological STQC-scenarios.
\end{abstract}

Keywords: geometric and condensed matter methods in modern cosmology; modified gravity theories; space-time quasiperiodic, aperiodic and quasicrystal cosmological structures; post modern inflation paradigm; accelerating cosmology; dark energy and dark matter.

MSC: 83C45, 83C99, 53D55, 53B40, 53B35

PACS: 47.54.-r; 05.45.-a; 47.35.Pq; 47.52.+j; 61.50.Ah; 61.44.Br; 05.20.-y; 64.70.D

\section{Contents}

\section{Introduction}

* The address for post correspondence: 140 Morehampton Rd, Donnybrook, Dublin 04, Ireland, D04 N2C0; emails: sergiu.vacaru@gmail.com ; sergiuvacaru@mail.fresnostate.edu 
2 Time quasi-periodic configurations in (modified) gravity 4

$2.12+2$ and $3+1$ splitting and distorted (non) linear connections . . . . . . . . . 4

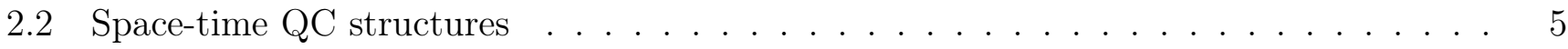

2.2.1 One dimensional relativistic time quasicrystal structures . . . . . . . . . . . 6

2.2.2 Two dimensional relativistic time quasicrystal structures . . . . . . . . . . 7

2.2.3 Three dimensional QC structures on curved spaces . . . . . . . . . . 8

2.2.4 Mixed 2-d TQC and 3-d QC configurations . . . . . . . . . . . . . 8

2.2.5 Matter sources and STQC structures in curved space-times . . . . . . . . . . 9

2.3 Gravitational and matter field equations for STQCs . . . . . . . . . . . . . . 10

2.4 Parameterizations for cosmological d-metrics . . . . . . . . . . . . . . . . 12

2.4.1 Target d-metrics with polarization functions . . . . . . . . . . 12

2.4.2 Parameterizations of prime d-metrics . . . . . . . . . . . . . 13

2.4.3 Approximations for target d-metrics . . . . . . . . . . . . . . . . . 14

3 Off-diagonal and quasi FLRW solutions with STQC structure 16

3.1 Cosmological ansatz for STQC structures . . . . . . . . . . . . . . . 16

3.2 Systems of nonlinear PDEs for cosmological STQCs . . . . . . . . . . . . . . 16

3.3 Generating functions and integrals for off-diagonal cosmological solutions . . . . . . 17

3.3.1 Extracting Levi-Civita STQC configurations . . . . . . . . . . . . . . . 19

3.3.2 Constructing d-metrics and N-connections from STQC generating functions . . 20

3.3.3 Off-diagonal quadratic line elements with nonholonomic STQC-torsion . . . . 21

3.3.4 Quadratic elements for quasi FLRW solutions encoding STQC structures ... 22

3.4 Examples of STQC, time QC and TC solutions . . . . . . . . . . . 23

3.4.1 Gravitational STQC structures generated by (effective) matter fields . . . . . 2 23

3.4.2 Interacting STQC gravitational and matter fields . . . . . . . . . . . . 24

3.4.3 A toy model with 1-TQC structure for DM . . . . . . . . . . . . 25

4 Inflationary STQC dynamics 25

4.1 General cosmological properties of gravity theories with STQC structure . . . . . . 26

4.1.1 Perfect fluid representation of STQC configurations in GR . . . . . . . . 2 26

4.1.2 Analogous perfect fluid representation of STQC structures in MGT . . . . . . 26

4.1.3 Non-minimal coupling of MGT and STQC configurations . . . . . . . . . 27

4.2 Effective scalar field and STQC inflation . . . . . . . . . . . . . 28

4.2.1 Scalar field description and gravitational STQC inflation . . . . . . . . 28

4.2.2 Inflation determined by a scalar field with TC structure . . . . . . . . . . 29

4.2 .3 STQC inflation from $F(R)$ theories . . . . . . . . . . . . . 30

4.2.4 Reheating and STQC structures in nonholonomic MGTs . . . . . . . . . 31

5 Late STQC dynamics, dark energy and dark matter 33

5.1 Quasi $\Lambda$ CDM epoch from STQC structures in $F(R)$ gravity $\ldots \ldots \ldots \ldots \ldots$

5.2 STQC unification of inflation with dark energy era in MGTs . . . . . . . . . . 34

6 Conclusions 35 


\section{Introduction}

This work provides a concise introduction into the cosmology with space-time quasi-periodic and pattern forming structures. For the latest developments on quasiperiodc cosmological models, we cite [1, 2, 3, 4, 5, 6] and references therein. The approach involves cosmological scenarios with locally anisotropic and inhomogenous gravitational and matter field interactions in general relativity, GR, and modified gravity theories MGTs. Such results on $f(R)$ cosmology and various generalizations are reviewed in [7, 8, 9, 10, 11, 12, 13, 14, 15, 16, 17. Hence we focus on cosmological models which can be described effectively by "quasi" Friedmann-Lamaître-Roberston-Walker, FLRW, metrics encoding space-time quasicrystal structures, STQC, modelled at cosmological scales. There will be written in brief and used such terms: quasicrystal, QC; time crystal, TC; time quasicrystal, TQC; time crystal, $\mathrm{TC}$, and similarly for corresponding nonlinear structures and space-time configurations described by solutions of classical or quantum equations not only in condensed matter physics but also in gravity theories, astrophysics and cosmology.

We show that nonlinear gravitational and matter field interactions define STQC structures which are quasiperiodic cosmological analogous to TC and QC. In our approach, we generalize the concepts of classical and quantum TCs developed in A. Shapere and F. Wilczek works [18, 19, 20, 21, , see references therein on other concepts of TC elaborated for biological systems, quantum coherence, and cosmology, and [22, 23] for a review and recent results. Here we note that two research groups were able already to create independently TCs in condensed matter physics [24, 25, 26, 27].

Let us explain in brief the difference and similarity between TC and QC structures in cosmology and condensed matter physics. Originally, A. Shapere and F. Wilczek [18] elaborated on classical TCs with properties which are time analogues of crystalline spatial order. They studied dynamical systems in the lowest energy state when within orbits of broken symmetry there are modelled interactions with traveling density waves. In a partner work [19], F. Wilczek proposed the existence of a new state of matter called "quantum time crystals", QTC, for quantum mechanical systems defined with a ground state displaying a time-dependent behaviour (periodic oscillation) of some physical observables. If normal crystals exhibit broken translation symmetry, the concept of TC was elaborated theoretically for classical and quantum many-body systems with self-organization in time. Such systems describe a periodic motion with possible jumps or periodic oscillations after a spontaneously breaking of time translation symmetry. The works on TCs and QTCs stimulated both a considerable interest and controversial debates. Several papers claiming that space-time crystals are impossible were published [28, 29]. In a series of subsequent works [30, 31], certain models of time translation symmetry breaking were elaborated and studied in details. That led to a conclusion that quantum TCs in equilibrium thermodynamical states are not possible [32]. As a consequence, several models of TCs which avoid the equilibrium no-go arguments were elaborated [21, 33], for instance, for time translation symmetry breaking of non-equilibrium systems [34, 35, 25].

The first goal of this paper is to prove that STQC, TQC and TC structures can be generated as (off-) diagonal locally anisotropic and inhomogeneous cosmological solutions in MGTs and GR. In particular, such (quasi) periodic gravitational and matter field structures are generated by a spontaneous broken gravitational vacuum metrics $g_{\alpha \beta}(\ldots, t)$ with dependencies on a time like coordinate $t$. We shall analyze subclasses of generating functions for off-diagonal metrics, or certain quasi FLRW configurations depending only on $t$, or modeling QC structures as in [5, 6]. For corresponding functional and parametric dependencies, such gravitational and matter field solutions encode data on STQC. The second our goal is to apply our geometric methods and new classes of cosmological so- 
lutions for elaborating new scenarios of inflation with (or induced by) STQC structure and analyse possible implications in dark energy and dark matter physics.

Our work is organized as follow. In section 2, we formulate the gravitational and matter field equations for STQCs in MGTs and GR. There are provided necessary parameterizations of cosmological metrics, coefficient formulas, geometric calculus and approximations. Section 3 is devoted to a geometric method of constructing exact cosmological solutions for STQC configurations in general locally anisotropic forms and/or with off-diagonal time dependence or quasi FLRW limits. The inflationary STQC dynamics is studied in section 4. Then, we consider models of late STQC dynamics and dark energy in section 5. Finally, conclusions are formulated in section 6.

\section{Time quasi-periodic configurations in (modified) gravity}

We outline certain advanced geometric methods [1, 2, 3, 4, 5, 6] which can be applied for modeling space-time configurations with STQC structure. Then, there are formulated basic equations defining space-time QCs and the gravitational and matter field equations for STQCs.

\section{$2.1 \quad 2+2$ and $3+1$ splitting and distorted (non) linear connections}

Let us consider a four dimensional, 4d, spacetime manifold $\mathbf{V}$ modelled as a Lorentz manifold enabled with a metric $\mathbf{g}$ of pseudo-Riemannian signature $(+++-)$. For a conventional $2+2 \mathrm{splitting}$, the local coordinates are denoted $u^{\gamma}=\left(x^{k}, y^{c}\right)$, or $u=(x, y)$, with indices $i, j, k, \ldots=1,2$ and $a, b, c, \ldots=3,4$. We can consider a $3+1$ splitting when $u^{4}=y^{4}=t$ is a time like coordinate and $u^{\grave{\imath}}=\left(x^{i}, y^{3}\right)$, where spacelike coordinates run values $\grave{i}, \grave{j}, \grave{k}, \ldots=1,2,3$. On respective tangent and cotangent Lorentz bundles, $T \mathbf{V}$ and (dual) $T^{*} \mathbf{V}$, the local bases/ frames are written as frame (equivalently, tetrad, or vierbein) transforms related to local coordinate bases, $e_{\alpha}=e_{\alpha}^{\alpha^{\prime}}(u) \partial_{\alpha^{\prime}}$, for $\partial_{\alpha^{\prime}}=\partial / \partial u^{\alpha^{\prime}}$, and cobases $e^{\alpha}=e^{\alpha}{ }_{\alpha^{\prime}}(u) d u^{\alpha^{\prime}}$.

A nonholonomic $2+2$ splitting is determined by a nonlinear connection, N-connection, structure $\mathbf{N}$ defined as a conventional horizontal, h, and vertical, $\mathrm{v}$, decomposition. It is stated by a respective nonholonomic (equivalently, anholonomic, i.e. nonintegrable) distribution,

$$
\mathbf{N}: \quad T \mathbf{V}=h T \mathbf{V} \oplus v T \mathbf{V}
$$

into respective h- and v-subspaces, $h T \mathbf{V}$ and $v T \mathbf{V} 1$ Any N-connection structure defines respective classes of $\mathrm{N}$-adapted (co) frames, $\mathbf{e}_{\alpha}=\left(\mathbf{e}_{i}, e_{a}\right) 2$ and dual frames, $\mathbf{e}^{\alpha}=\left(x^{i}, \mathbf{e}^{a}\right)$,

$$
\begin{aligned}
\mathbf{e}_{i} & =\partial / \partial x^{i}-N_{i}^{a}(u) \partial / \partial y^{a}, e_{a}=\partial_{a}=\partial / \partial y^{a}, \\
e^{i} & =d x^{i}, \mathbf{e}^{a}=d y^{a}+N_{i}^{a}\left(u^{\gamma}\right) d x^{i} .
\end{aligned}
$$

Any (pseudo) Riemannian metric on $\mathbf{V}$ can be parameterized as a distinguished metric, $\mathrm{d}-$ metric,

$$
\begin{aligned}
\mathbf{g} & =\mathbf{g}_{\alpha \beta}(u) \mathbf{e}^{\alpha} \otimes \mathbf{e}^{\beta}=g_{i}\left(x^{k}\right) d x^{i} \otimes d x^{i}+h_{a}\left(x^{k}, y^{b}\right) \mathbf{e}^{a} \otimes \mathbf{e}^{b} \\
& =\mathbf{g}_{\alpha^{\prime} \beta^{\prime}}(u) \mathbf{e}^{\alpha^{\prime}} \otimes \mathbf{e}^{\beta^{\prime}}, \text { for } \mathbf{g}_{\alpha^{\prime} \beta^{\prime}}(u)=\mathbf{g}_{\alpha \beta} \mathbf{e}_{\alpha^{\prime}}^{\alpha} \mathbf{e}_{\beta^{\prime}} .
\end{aligned}
$$

\footnotetext{
${ }^{1}$ Such formulas can be written in local form, $\mathbf{N}=N_{i}^{a} \frac{\partial}{\partial y^{a}} \otimes d x^{i}$, using the N-connection coefficients $N_{i}^{a}$; we shall use the Einstein convention on summation on up-low repeating indices if the contrary will not be stated; the geometric objects on $\mathbf{V}$ will be labeled by "bold face" symbols if they can be written in N-adapted form.

${ }^{2}$ a local basis $\mathbf{e}_{\alpha}$ is nonholonomic if the commutators $\mathbf{e}_{[\alpha} \mathbf{e}_{\beta]}:=\mathbf{e}_{\alpha} \mathbf{e}_{\beta}-\mathbf{e}_{\beta} \mathbf{e}_{\alpha}=C_{\alpha \beta}^{\gamma}(u) \mathbf{e}_{\gamma}$ contain nontrivial anholonomy coefficients $C_{\alpha \beta}^{\gamma}=\left\{C_{i a}^{b}=\partial_{a} N_{i}^{b}, C_{j i}^{a}=\mathbf{e}_{j} N_{i}^{a}-\mathbf{e}_{i} N_{j}^{a}\right\}$
} 
A distinguished connection, d-connection, (equivalently, a d-covariant derivative) $\mathbf{D}=\left\{\mathbf{D}_{\alpha}\right\}=$ $\left(h D=\left\{D_{i}\right\}, v D=\left\{D_{a}\right\}\right)$, is defined as a linear connection, preserving the $\mathrm{N}$-connection splitting $\mathbf{N}$ (11) under parallel transports. The d-connection coefficients $\mathbf{D}=\left\{\boldsymbol{\Gamma}_{\beta \gamma}^{\alpha}=\left(L^{i}{ }_{j k}, \dot{L}^{a}{ }_{b k} ; \dot{C}^{i}{ }_{j c}, C^{a}{ }_{b c}\right)\right\}$, with respective h- and v-covariant derivatives, ${ }_{h} \mathbf{D}=\left\{\left(L^{i}{ }_{j k}, \dot{L}^{a}{ }_{b k}\right)\right\}$ and ${ }_{v} \mathbf{D}=\left\{\left(\dot{C}^{i}{ }_{j c}, C^{a}{ }_{b c}\right)\right\}$, are computed in $\mathrm{N}$-adapted form with respect to frames (2).

A nonholonomic metric-affine Lorentz manifold is defined by data $(\mathbf{V}, \mathbf{N}, \mathbf{D}, \mathbf{g})$, where $\mathbf{g}$ is a Lorentz metric. In general, a d-connection $\mathbf{D}=\nabla+\mathbf{Z}$, with a distortion tensor $\mathbf{Z}$, is different from the standard Levi-Civita connection, LC-connection, $\nabla$, which (by definition) is metric compatible and with zero torsion 3

On a nonholonomic Lorentz manifold $(\mathbf{V}, \mathbf{N})$, we can work equivalently with two important linear connections:

$$
(\mathbf{g}, \mathbf{N}) \rightarrow\left\{\begin{array}{c}
\nabla: \quad \nabla \mathbf{g}=0 ; \nabla \mathcal{T}=0, \text { for the Levi-Civita, LC, -connection } \\
\widehat{\mathbf{D}}: \quad \widehat{\mathbf{D}} \mathbf{g}=0 ; h \widehat{\mathcal{T}}=0, v \widehat{\mathcal{T}}=0, h v \widehat{\mathcal{T}} \neq 0, \text { for the canonical d-connection }
\end{array}\right.
$$

We note that both $\nabla$ and $\widehat{\mathbf{D}}$ are defined by the same metric structure $\mathbf{g}$ which allows to model any (pseudo) Riemannian geometry as an effective a (pseudo) Riemann-Cartan geometry with nonholonomically induced torsion structure $\widehat{\mathcal{T}}=\left\{\widehat{\mathbf{T}}_{\beta \gamma}^{\alpha}\left[N_{i}^{a}, \mathbf{g}_{\beta \gamma}\right]\right\}$. The coefficients $\widehat{\mathbf{T}}_{\beta \gamma}^{\alpha}$ are determined by nontrivial anholonomy coefficients $C_{\alpha \beta}^{\gamma}$ and the coefficients of the metric and N-connection. It is always possible to imposing additional nonholonomic constraints, or limits, when, $\widehat{\mathcal{T}}=0$ and $\widehat{\mathbf{D}}_{\mid \widehat{\mathcal{T}}=0}=\nabla \underline{4}$

The canonical d-connection $\widehat{\mathbf{D}}$ is very important because it allows a general decoupling and integration of modified Einstein equations. In our approach we consider systems of nonlinear partial differential equations, PDEs, in general forms and do not reduce them to some particular cases of systems of nonlinear ordinary differential equations, ODEs. We work with various classes of off-diagonal metrics $\mathbf{g}_{\beta \gamma}\left(x^{k}, y^{c}\right)$ depending, in principle, on all spacetime coordinates, see details in [6] and references therein. Having found a class of solutions, for instance, for locally anisotropic cosmological metrics $\left.\mathbf{g}_{\beta \gamma}\left(x^{k}, t\right)\right)$ determined by respective sets of generating and integration functions, we can impose additional constraints which allow us to extract LC-configurations with $\widehat{\mathbf{D}}_{\mid \widehat{\mathcal{T}}=0}=\nabla$ and (as particlar cases) cosmological solutions with $\mathbf{g}_{\beta \gamma}(t)$. Generalized (off-)diagonal classes of solutions (for instance, with STQC structure) can not be constructed if we work from the beginning with $\nabla$ for some high symmetric ansatz for metrics - this is a general property of the systems of nonlinear PDEs.

\section{$2.2 \quad$ Space-time QC structures}

In this subsection, we provide three examples how time quasiperiodic structures can be defined in a curved space-time. We consider one and two dimensional TQCs with time crystal equations

\footnotetext{
${ }^{3}$ Using standard methods of differential geometry, we can define and compute the N-adapted coefficients of the d-tensors for curvature $\mathcal{R}=\left\{\mathbf{R}_{\beta \gamma \delta}^{\alpha}\right\}$, torsion $\mathcal{T}=\left\{\mathbf{T}_{\beta \gamma}^{\alpha}\right\}$, and nonmetricity $\mathcal{Q}=\left\{\mathbf{Q}_{\alpha \beta \gamma}:=\mathbf{D}_{\alpha} \mathbf{g}_{\beta \gamma}\right\}$.

${ }^{4}$ The curvature tensors of both linear connections are computed in standard forms, respectively, for $\widehat{\mathbf{D}}$ and $\nabla$, when $\widehat{\mathcal{R}}=\left\{\widehat{\mathbf{R}}_{\beta \gamma \delta}^{\alpha}\right\}$ and $\nabla_{\mathcal{R}}=\left\{R_{\beta \gamma \delta}^{\alpha}\right\}$. The corresponding Ricci tensors are $\widehat{\mathcal{R}} i c=\left\{\widehat{\mathbf{R}}_{\beta \gamma}:=\widehat{\mathbf{R}}_{\alpha \beta \gamma}^{\gamma}\right\}$ and $\operatorname{Ric}=\left\{R{ }_{\beta \gamma}:=\right.$ $\left.R_{\alpha \beta \gamma}^{\gamma}\right\}$, where $\widehat{\mathcal{R}} i c$ is characterized by $h-v$ N-adapted coefficients, $\widehat{\mathbf{R}}_{\alpha \beta}=\left\{\widehat{R}_{i j}:=\widehat{R}_{i j k}^{k}, \widehat{R}_{i a}:=-\widehat{R}_{i k a}^{k}, \widehat{R}_{a i}:=\right.$ $\left.\widehat{R}_{a i b}^{b}, \widehat{R}_{a b}:=\widehat{R}_{a b c}^{c}\right\}$. There are also two different scalar curvatures, $R:=\mathbf{g}^{\alpha \beta} R_{\alpha \beta}$ and $\widehat{\mathbf{R}}:=\mathbf{g}^{\alpha \beta} \widehat{\mathbf{R}}_{\alpha \beta}=g^{i j} \widehat{R}_{i j}+$ $g^{a b} \widehat{R}_{a b}$.
} 
generalizing those introduced in [22]); three dimensional QC structures studied in [6, 5]; and mixed configurations resulting in STQC. We emphasize that in this work we use a different system of notation for partial derivatives when, for instance, $\partial q / \partial x^{i}=\partial_{i} q, \partial q / \partial y^{3}=\partial_{3} q$, and $\partial q / \partial y^{4}=$ $\partial_{4} q=\partial_{4} q=q^{\bullet}$, for a function $q\left(x^{i}, y^{3}, t\right)$. A prime will be used for any functional derivative like $F^{\prime}(R)=d F / d R$, for instance, in a modified gravity with $F(R)$, where is a Ricci scalar.

\subsubsection{One dimensional relativistic time quasicrystal structures}

Such a structure on a space-time $(\mathbf{V}, \mathbf{g}, \mathbf{N})$ can be modelled by a scalar field $\varsigma\left(x^{i}, y^{a}\right)$ and a Lagrange density

$$
\dot{L}\left[\varsigma\left(x^{i}, y^{a}\right)\right]=\frac{1}{48}\left(\mathbf{g}^{\alpha \beta}\left(\mathbf{e}_{\alpha} \varsigma\right)\left(\mathbf{e}_{\beta} \varsigma\right)\right)^{2}-\frac{1}{4} \mathbf{g}^{\alpha \beta}\left(\mathbf{e}_{\alpha} \varsigma\right)\left(\mathbf{e}_{\beta} \varsigma\right)-V^{\prime}(\varsigma)
$$

where $V^{\prime}(\varsigma)$ is a nonlinear potential and $\mathbf{e}_{\alpha}$ (1) are N-adapted partial derivatives. This $\dot{L}$ results in variational motion equations of type

$$
\left[\frac{1}{2} \mathbf{g}^{\alpha \beta}\left(\mathbf{e}_{\alpha} \varsigma\right)\left(\mathbf{e}_{\beta} \varsigma\right)-1\right]\left(\widehat{\mathbf{D}}^{\gamma} \widehat{\mathbf{D}}_{\gamma} \varsigma\right)=2 \frac{\partial \dot{V}}{\partial \varsigma} .
$$

We say that a field $\varsigma$ defines a one dimensional (1-d) time quasicrystal structure, 1-TQC, if it is a solution of the equation ([6).

For non-relativistic approximations with $g_{\alpha \beta}=[1,1,1,-1]$ and $\varsigma \rightarrow \varsigma(t)$, we obtain an effective Lagrange density

$$
\dot{L}\left[\varsigma\left(x^{i}, y^{a}\right)\right] \rightarrow \dot{L}[\varsigma(t)]=\frac{1}{12}\left(\varsigma^{\bullet}\right)^{4}-\frac{1}{2}\left(\varsigma^{\bullet}\right)^{2}-\dot{V}^{\prime}(\varsigma),
$$

leading to an effective energy $E=\frac{1}{4}\left[\left(\varsigma^{\bullet}\right)^{2}-1\right]^{2}+\hat{V}^{\prime}(\varsigma)-\frac{1}{4}$, and motion equations

$$
\left[\left(\varsigma^{\bullet}\right)^{2}-1\right] \varsigma^{\bullet \bullet}=-\frac{\partial \dot{V}^{\prime}}{\partial \varsigma} \text {. }
$$

Such equations were introduced in [22] for an example of 1-dTC-structure in condensed matter physics. The equations (66) provide a generalization for 1-TQCs modeled on a curved space-time.

A Lagrange density of type $L[\varsigma(t)]$ (17) may describe a simplest example of 1-dTC-structure in cosmology theories if such a value can be obtained effectively from a $L\left[\varsigma\left(x^{i}, y^{a}\right)\right]$ (5) related to certain gravitational Lagrange densities (for instance, describing dark energy, DE, configurations) and/or matter sources (for corresponding dark matter, DM, models). Such cosmological TC-structures are defined by some exact or parametric solutions of gravitational field equations in a MGT, or in GR. In general, such solutions are for metrics and (effective) matter fields depending both on time and space coordinates which allows us to describe more realistically inhomogeneous and/or locally anisotropic interactions of gravitational, DM and standard matter fields. We shall consider details on elaborating such cosmological models in next sections. Here, we note that 1-dTC-structures with time depending $\dot{L}[\varsigma(t)]$ (7) can be extracted by additional assumptions/ constraints from certain classes of generic offdiagonal solutions involving a $L_{\varsigma}\left[\varsigma\left(x^{i}, y^{a}\right)\right]($ (5) $)$. We loose the possibility to generate 1-dTC-structures in general forms if we work from the very beginning only with $L[\varsigma(t)]$ (before constructing a class of solutions with metrics and fields depending on $\left.\left(x^{i}, y^{a}\right)\right)$. This is the property of nonlinear dynamics and respective systems of nonlinear partial differential equations PDEs (see below formulas (18) and discussion therein). 


\subsubsection{Two dimensional relativistic time quasicrystal structures}

We can consider also a 2-d time quasicrystal structure, 2-TQC, defined by two coupled scalar fields $\chi\left(x^{i}, y^{a}\right)$ and $\breve{q}\left(x^{i}, y^{a}\right)$, for a model with effective planar charge in certain effective external fields on a curved spacetime. The Lagrangian is postulated

$$
\breve{L}\left(\chi\left(x^{i}, y^{a}\right), q\left(x^{i}, y^{a}\right)\right)=\frac{\chi_{0}}{4}\left(\mathbf{g}^{\alpha \beta}\left(\mathbf{e}_{\alpha} \chi\right)\left(\mathbf{e}_{\beta} \chi\right)\right)^{2}+\breve{B}^{\gamma}(\chi) \mathbf{e}_{\gamma} \breve{q}-\breve{Q}(\chi)-\breve{V}(\breve{q})
$$

where the constant $\chi_{0}$, functionals $B^{\gamma}(\chi)$ and $Q(\chi)$, and effective potential $\breve{V}(\breve{q})$ define a corresponding time quasiperiodic structure. The resulting variotional motion equations are

$$
\begin{aligned}
\chi_{0} \widehat{\mathbf{D}}^{\gamma} \widehat{\mathbf{D}}_{\gamma} \chi & =-\frac{\partial \breve{B^{\gamma}}(\chi)}{\partial \chi} \mathbf{e}_{\gamma} \breve{q}+\frac{\partial \breve{Q}(\chi)}{\partial \chi} \\
\frac{\partial \breve{B}^{\gamma}(\chi)}{\partial \chi} \mathbf{e}_{\gamma} \chi & =-\frac{\partial \breve{V}}{\partial \breve{q}} .
\end{aligned}
$$

In a non-relativistic limit with time depending scalar fields $\chi(t)$ and $q(t)$, we obtain

$$
\breve{L}\left(\chi\left(x^{i}, y^{a}\right), q\left(x^{i}, y^{a}\right)\right) \rightarrow \breve{L}(\chi(t), q(t))=\frac{\chi_{0}}{2}\left(\chi^{\bullet}\right)^{2}+\breve{B}^{4}(\chi) q^{\bullet}-\breve{Q}(\chi)-\breve{V}(\breve{q})
$$

and the motion equations

$$
\begin{aligned}
\chi_{0} \chi^{\bullet \bullet} & =\frac{\partial \breve{B}(\chi)}{\partial \chi} \breve{q}^{\bullet}-\frac{\partial \breve{Q}(\chi)}{\partial \chi} \\
\chi^{\bullet} \frac{\partial \breve{B}(\chi)}{\partial \chi} & =-\frac{\partial \breve{V}}{\partial \breve{q}},
\end{aligned}
$$

which describe an effective dynamics considered to 2-d TC in [22]. For additional constraints, such an effective 2-d dynamics reproduces 1 -d TC dynamics with an effective small positive mass $\chi_{0}$ acting as a regulator for the 1-d time crystal dynamical system.

In this work, our goal is to provide a relativistic curve space-time generalization of the so-called sisyphus dynamics with microstructure and ratcheting is possible if we consider effective Lagrange densities $\breve{L}\left(\chi\left(x^{i}, y^{a}\right), q\left(x^{i}, y^{a}\right)\right)$ (8) for 2-TQC structures. Certain such configurations contain as particular cases 1-dTC-structure but the extensions of nonlinear dynamics from lower to higher dimensions is not trivial. For instance, we can use such $\breve{L}$ to model DM contributions via an effective energy momentum tensor $\overline{\mathbf{T}}_{\alpha \beta}$ computed for $\overline{\mathcal{L}}=\breve{L}$, see below formula (17). Exact and parametric cosmological solutions with DM 2-TQC sources can be constructed for generalized Einstein equations (see below the formulas (18) and, for respective energy-momentum tensor, (21)) as it is described in section 3. We can model cosmological 2-TQC structures depending only on a time like coordinate by considering certain limits/ constraints when $\breve{L} \rightarrow \breve{L}(\chi(t), q(t))$ (10). Finally, it should be emphasized that we can generate pure gravitational 2-TQC structures if a $\breve{L}$ is used for constructing a gravitational Lagrange density in a MGT or GR. This can be used for modelling nonlinear vacuum gravitational interactions and/or DE effects, see below discussions related to formula (13). 


\subsubsection{Three dimensional QC structures on curved spaces}

Quasiperiodic structures can be modeled as spacelike configurations on curved spacetimes (called also quasicrystals, QG), see details in [6, 5]. Let us consider a $3+1$ decomposition with space like coordinates $x^{\grave{\imath}}$ (for $\grave{\imath}=1,2,3$ ), time like coordinate $y^{4}=t$, which is adapted to another $2+2$ decomposition with fibration by 3 -d hypersurfaces $\widehat{\Xi}_{t}$. We define a canonically nonholonomically deformed Laplace operator ${ }^{b} \widehat{\Delta}:=\left({ }^{b} \widehat{D}\right)^{2}=b^{i \grave{j}} \widehat{D}_{i} \widehat{D}_{\grave{j}}$, for the 3 -d part of a d-metric. The distortion of ${ }^{b} \Delta:=\left({ }^{b} \nabla\right)^{2}$ can be defined on any $\widehat{\Xi}_{t}$ using a d-metric (3) and respective restrictions of $\widehat{\mathbf{D}}$.

For instance, a QC structure can be defined by a scalar field $\psi\left(x^{i}, y^{a}\right)$ which is a solution of an evolution equation with conserved dynamics of type

$$
\psi^{\bullet}={ }^{b} \widehat{\Delta}\left[\frac{\delta \breve{F}}{\delta \psi}\right]=-{ }^{b} \widehat{\Delta}\left(\Theta \psi+Q \psi^{2}-\psi^{3}\right)
$$

The functional $\breve{F}$ in (11) defines an effective free energy

$$
\breve{F}[\psi]=\int\left[-\frac{1}{2} \psi \Theta \psi-\frac{Q}{3} \psi^{3}+\frac{1}{4} \psi^{4}\right] \sqrt{b} d x^{1} d x^{2} \delta y^{3},
$$

where $b=\operatorname{det}\left|b_{i j}\right|, \delta y^{3}=\mathbf{e}^{3}$ and the operator $\Theta$ and parameter $Q$ are defined in the partner work [6. 5]. Values $\Theta$ and $Q$ determine certain quasiperiodic, aperiodic and/or $\mathrm{QC}$ order on curved spacetimes.

In this work, we show that cosmological solutions with QC structure can be extended to more general classes with TQC. This is possible if we generalize functionals $\breve{F}$ in (11) in some forms including additional dependencies, for instance, on effective Lagrange densities $\breve{L}$ (8) for 2-TQC structures. In next subsection we consider an explicit such example.

\subsubsection{Mixed 2-d TQC and 3-d QC configurations}

We can model on $(\mathbf{V}, \mathbf{g}, \mathbf{N})$ nonholonomic configurations both with TQC and QC structure $(\chi, q)$. Such a model can be generated by a function $\chi$ as in (9) when a function $q$ is additionally subjected to a QC-evolution condition, when

$$
\begin{aligned}
q^{\bullet} & ={ }^{b} \widehat{\Delta}\left[\frac{\delta \dot{F}}{\delta q}\right]=-{ }^{b} \widehat{\Delta}\left(\dot{\Theta} q+\dot{Q} q^{2}-q^{3}\right) \text { for } \\
F^{\prime}[\chi, q] & =\int\left[-\frac{1}{2} q \dot{\Theta} q-\frac{\dot{Q}}{3} q^{3}+\frac{1}{4} q^{4}\right] \sqrt{b} d x^{1} d x^{2} \delta y^{3} .
\end{aligned}
$$

In principle, we can generate more complex STQC structures when certain QC conditions are imposed additionally for $\chi$. Here we note that we wrote $\breve{F}$ in (11) and $\dot{F}$ (12) in order to avoid future ambiguities with conventions for MGT with $\mathbf{F}(\widehat{\mathbf{R}})$, see below formula (20).

The scalar fields $(\chi, q)$ subjected to the conditions of evolution (12) can be included into certain effective Lagrange densities $\breve{L}(8)$ and respective energy momentum tensor $\overline{\mathbf{T}}_{\alpha \beta}$ computed for $\overline{\mathcal{L}}=\breve{L}$ (17). In result, we can elaborate on cosmological models with DM and DE evolution encoding both TQC and QC structures, see sections 4 and 5 . 


\subsubsection{Matter sources and STQC structures in curved space-times}

There are two possibilities to generate STQC structures in gravity theories:

1. When some coefficients of metrics and/or (non) linear connections with functional dependencies on STQC generating functions: Such configurations will be defined by certain data $\varsigma=\bar{\varsigma}\left(x^{i}, y^{a}\right)$, see (6); $\chi=\bar{\chi}\left(x^{i}, y^{a}\right)$ and $\breve{q}=\bar{q}\left(x^{i}, y^{a}\right)$, see (9); $\psi=\bar{\psi}\left(x^{i}, y^{a}\right)$, see (11); and/or $\chi=\bar{\chi}\left(x^{i}, y^{a}\right)$ and $q=\bar{q}\left(x^{i}, y^{a}\right)$, see (12), contained in a functional form for a d-metric (3)

$$
\mathbf{g}_{\alpha \beta}=\overline{\mathbf{g}}_{\alpha \beta}\left[x^{i}, y^{a} ; \bar{\varsigma}, \bar{\chi}, \bar{q}, \bar{\psi}, \bar{q}, \ldots\right],
$$

where symbols are overlined in order to emphasize that such values are considered for geometric objects defining tensor and/or connection fields in a MGT or GR. In result, functional dependencies on $\bar{\zeta}, \bar{\chi}, \bar{q}, \bar{q}, \ldots$ (dots are used for any other possible sets of generating functions, for instance, solitonic waves, other QC structures etc) can be computed for (non) linear connections, $N_{i}^{a}=\bar{N}_{i}^{a}\left[x^{i}, y^{a} ; \bar{\varsigma}, \bar{\chi}, \bar{q}, \bar{\psi}, \bar{q}, \ldots\right]$ and $\boldsymbol{\Gamma}_{\beta \gamma}^{\alpha}=\overline{\boldsymbol{\Gamma}}_{\beta \gamma}^{\alpha}\left[x^{i}, y^{a} ; \bar{\varsigma}, \bar{\chi}, \bar{q}, \bar{\psi}, \bar{q}, \ldots\right] ;$ respective curvatures, torsions etc. computed, for instance, for $\nabla[\overline{\mathbf{g}}]$ and/or $\widehat{\mathbf{D}}[\overline{\mathbf{g}}]$.

In a series of our previous works [1, 3, 6] and references therein, we studied cosmological and black hole metrics which are similar to (13) if they are not stationary but extended to configurations with evolution and encoding possible QC structures. The main goal of this article is prove that the AFDM allows constructions with STQC structures for elaborating more realistic cosmological DE and DM models. In explicit form, we consider examples with 1-TQC and 2-TQC configurations.

2. By matter fields as STQC sources: For simplicity, we can consider actions

$$
{ }^{m} \mathcal{S}=\int d^{4} u \sqrt{|\mathbf{g}|}^{m} \mathcal{L}
$$

for matter field Lagrange densities ${ }^{m} \mathcal{L}(\phi)$ [we label by $\phi\left(x^{i}, y^{a}\right)$ all necessary sets of matter fields from standard particle physics and GR] depending only on coefficients of a metric field and do not depend on their derivatives when

$$
{ }^{m} \mathbf{T}_{\alpha \beta}:=-\frac{2}{\sqrt{\left|\mathbf{g}_{\mu \nu}\right|}} \frac{\delta\left(\sqrt{\left|\mathbf{g}_{\mu \nu}\right|}{ }^{m} \mathcal{L}\right)}{\delta \mathbf{g}^{\alpha \beta}}={ }^{m} \mathcal{L} \mathbf{g}^{\alpha \beta}+2 \frac{\delta\left({ }^{m} \mathcal{L}\right)}{\delta \mathbf{g}_{\alpha \beta}} .
$$

Additionally, we can consider Lagrange densities for 1-d, 2-d TQC, with additional prescriptions for QC like structures as we used above for deriving the motion equations (6), (9), and/or (12). The sum of such Lagrange densities is written

$$
\overline{\mathcal{L}}[\varsigma, \chi, q, \psi, \breve{q}, \ldots]=\dot{L}(\varsigma)+\breve{L}(\chi, q)+\ldots
$$

which allows us to compute the energy-momentum tensor for STQC-matter,

$$
\overline{\mathbf{T}}_{\alpha \beta}:=-\frac{2}{\sqrt{\left|\mathbf{g}_{\mu \nu}\right|}} \frac{\delta\left(\sqrt{\left|\mathbf{g}_{\mu \nu}\right|} \overline{\mathcal{L}}\right)}{\delta \mathbf{g}^{\alpha \beta}} .
$$

A source $\overline{\mathbf{\Upsilon}}_{\mu \nu}=M_{P}^{-2}\left(\overline{\mathbf{T}}_{\mu \nu}-\frac{1}{2} \mathbf{g}_{\mu \nu} \overline{\mathbf{T}}_{\mu \nu}\right)$, with $\overline{\mathbf{T}}=\overline{\mathbf{T}}_{\mu \nu} \mathbf{g}^{\mu \nu}$ and $M_{P}$ being the Planck mass determined by the gravitational constant, can be used for modelling DM effects with pattern forming and STQCstructure. 
Let us clarify the link between (effective) Lagrangians and functionals for STQC structures considered in this section and the rest of the paper: Observational data for modern accelerating cosmology and DE and DM physics provide evidence that our Universe has a very complex nonlinear evolution structure. For different space and time scales, the meta galactic structure and dynamic can be conventionally described by certain STQC and/or QC configurations with quasi periodic inhomogeneities and anisotropies, nonlinear fluctuations etc. In realistic forms, such configurations can be modelled by effective dynamical and evolution theories determined by sums of Lagrange densities $\overline{\mathcal{L}}=\dot{L}(\varsigma)+\breve{L}(\chi, q)+\ldots(16)$ including, for instance, terms for generalized 1- and/or 2-TQC structures, see formulas (15) and/or (8) .

The goal of this work is to show that cosmological models with STQC structure can be defined by exact or parametric solutions of the gravitational field equations in MGT and/or GR. In such an approach, the effective fields modelling STQC configurations with a $\overline{\mathcal{L}}$ have to be encoded into a metric structure $\overline{\mathrm{g}}_{\alpha \beta}\left[x^{i}, y^{a} ; \bar{\varsigma}, \bar{\chi}, \bar{q}, \bar{\psi}, \bar{q}, \ldots\right]$ (13), and related (non) linear connections, and/or an energy-momentum tensor for STQC-matter $\overline{\mathbf{T}}_{\alpha \beta}$ (17) as we explained above. We develop also a geometric method for generating solutions of (modified) Einstein equations which model gravitational and (effective) matter field interactions with TC like and generalized STQC structure, see reviews of results in [1, 3, 6]. In our previous works [5, 6], quasiperiodic configurations where studied for space like QC structures. In section 3, we show that the anholonomic frame deformation method, ADFM, provides also an analytic techniques for generating STQC cosmological solutions. There are used new classes of generic off-diagonal ansatz, and considered their diagonalization, in section 2.4 .

Examples of STQC, time QC and TC solutions are provided in explicit form in section 3.4. The simplest (toy) example involving cosmological solutions with 1-TQC structure when the energy momentum tensor $\overline{\mathbf{T}}_{\alpha \beta}[\dot{L}(\varsigma)]$ (17) modelling DM is computed for $\overline{\mathcal{L}}=\dot{L}(\varsigma)$ (5) is analyzed in brief in subsection 3.4.3. Here we note that even such very simplified nonlinear evolution cosmological models with 1-TQC are described by quite sophisticate formulas because time and space like quasiperiodicity involves more general classes of generating functions and effective scalar fields.

Another very important relations of (effective) Lagrangians and functionals for time and space like (quasi) periodic configurations are stated in section 4 (contributing substantially in different models of STQC) and 5 (a late STQC cosmological dynamics with DE and DM interactions). We prove that in all such (modified) gravitational theories, effective Lagrange densities of type $L(\varsigma)$ (15) and $\breve{L}(\chi, q)$ (8) and functionals of type $\breve{F}[\psi]$ and $F[\chi, q]$, see respective formulas (11) and (12), describe more realistically the cosmological structure and evolution of our Universe.

\subsection{Gravitational and matter field equations for STQCs}

The motion equations for a MGT with a functional $\mathbf{F}(\widehat{\mathbf{R}})$ for the Lagrange density for gravitational field can be derived by a N-adapted variational calculus, see details in [1, 2, 3, 4, 4, 6, 6] and references therein 5 Such a system of nonlinear PDEs can be represented in an effective Einstein form,

$$
\widehat{\mathbf{R}}_{\mu \nu}=\Upsilon_{\mu \nu} .
$$

with sources in the right side parameterized in the form

$$
\Upsilon_{\mu \nu}={ }^{F} \Upsilon_{\mu \nu}+{ }^{m} \Upsilon_{\mu \nu}+\bar{\Upsilon}_{\mu \nu}
$$

\footnotetext{
${ }^{5}$ The geometric construction are similar to those for $f(R)$ gravity and various generalizations [7, 8, 14, 16, 17, but generalized for nonholonomic manifolds with $\mathrm{N}$-connection splitting (1).
} 
In these formulas, the effective energy-momentum tensor

$$
F \Upsilon_{\mu \nu}=\left(\frac{\mathbf{F}}{2 \mathbf{F}^{\prime}}-\frac{\widehat{\mathbf{D}}^{2} \mathbf{F}^{\prime}}{\mathbf{F}^{\prime}}\right) \mathbf{g}_{\mu \nu}+\frac{\widehat{\mathbf{D}}_{\mu} \widehat{\mathbf{D}}_{\nu} \mathbf{F}^{\prime}}{\mathbf{F}^{\prime}}
$$

is determined by the functional $\mathbf{F}(\widehat{\mathbf{R}})$ determines an effective energy-momentum tensor.

We can always define nonholonomic variable for a large class of commutative and noncommutative MGTs (including contributions, for superstring and/or supergravity models, metric-affine gravity with nonmetricity and torsion etc) when the modified (generalized) gravitational field equations can be represented in the form (18). Corresponding effective and matter field sources (19) may encode various contributions from extra dimensions, distortions of connection structures, super symmetric and noncommutative terms etc. For simplicity, in this work we consider only effective sources ${ }^{F} \boldsymbol{\Upsilon}_{\mu \nu}$ (18) determined by $f(R)$-theories but generalized to distortions of linear connections of type $\widehat{\mathbf{D}}=$ $\nabla+\widehat{\mathbf{Z}}$, with a distortion d-tensor $\widehat{\mathbf{Z}}$ uniquely defined by formula (44). As we proved in our above cited works on the AFDM and applications, it is convenient to use the the canonical d-connection $\widehat{\mathrm{D}}$ when formula written for a special class of $\mathrm{N}$-adapted frames allow certain general decoupling and integration of generalized Einstein equations (18). Such general decoupling with generic off-diagonal metrics depending, in principle, on all spacetime coordinates via certain classes of generating and integration functions and generating sources is not possible if we work only with the Levi-Civita connection $\nabla$.

The source for the matter fields and STQC matter configurations (they can be for standard matter fields in GR, or for certain DM models, with supgravity/noncommutative additional terms etc.) can be computed in standard form,

$$
{ }^{m} \Upsilon_{\mu \nu}=\frac{1}{2 M_{P}^{2}}{ }^{m} \mathbf{T}_{\mu \nu} \text { amd } \bar{\Upsilon}_{\mu \nu}=\frac{1}{2 M_{P}^{2}} \overline{\mathbf{T}}_{\mu \nu}
$$

Such (effective) energy-momentum tensors can be postulated in a geometric form like in GR (but for generalized connections) and/or derived equivalently following a $\mathrm{N}$-adapted gravitationsl calculus. They are included as respective terms for a generalized source $\Upsilon_{\mu \nu}(19)$, when $\bar{\Upsilon}_{\mu \nu}$ is determined by the energy-momentum of certain STQC like matter fields. Such quasiperiodic time and space structures can be studied by the same geometric methods both in GR and MGTs. In this work we consider generalized constructions for $\mathbf{F}(\widehat{\mathbf{R}})$-gravity because such theories are intensively studied for elaborating models of acclerating cosmology and DE and DM theories. It is also important to show that the AFDM can be applied for generating solutions with STQC structure in the bulk of modern gravity theories.

In our previous works [1, 2, 3, 6], we proved that we can construct exact solutions for the system (18) in explicit form for any source (19) which via frame transforms $\Upsilon_{\mu \nu}=e_{\mu}^{\mu^{\prime}} e^{\nu^{\prime}} \boldsymbol{\Upsilon}_{\mu^{\prime} \nu^{\prime}}$ can be parameterized into $\mathrm{N}$-adapted diagonalized form

$$
\Upsilon^{\mu}{ }_{\nu}=\operatorname{diag}\left[{ }_{h} \Upsilon\left(x^{i}\right),{ }_{h} \Upsilon\left(x^{i}\right), \Upsilon\left(x^{i}, t\right), \boldsymbol{\Upsilon}\left(x^{i}, t\right)\right]
$$

In these formulas, the values

$$
{ }_{h} \Upsilon\left(x^{i}\right)={ }_{h}^{F} \Upsilon\left(x^{i}\right)+{ }_{h}^{m} \Upsilon\left(x^{i}\right)+{ }_{h} \bar{\Upsilon}\left(x^{i}\right) \text { and } \Upsilon\left(x^{i}, t\right)={ }^{F} \Upsilon\left(x^{i}, t\right)+{ }^{m} \Upsilon\left(x^{i}, t\right)+\bar{\Upsilon}\left(x^{i}, t\right)
$$

are considered as generating source functions which impose certain nonholonomic constraints on the dynamics of (effective) matter fields. Having constructed a class of exact/parametric solutions 
we have to prescribe (23) in some forms which an explicit solution will describe/explain observational/experimental data. 6

Finally, we note that the Einstein equations for GR are obtained as a particular case if $\mathbf{F}(\widehat{\mathbf{R}})=R$ with a parametrization when ${ }^{F} \boldsymbol{\Upsilon}_{\mu \nu}[R]=0$ for $\widehat{\mathbf{D}}_{\mid \widehat{\mathcal{T}} \rightarrow 0}=\nabla$. Nontrivial STQC sources $\overline{\boldsymbol{\Upsilon}}_{\nu}^{\mu}=$ $\operatorname{diag}\left[{ }_{h} \bar{\Upsilon}\left(x^{i}\right),{ }_{h} \bar{\Upsilon}\left(x^{i}\right), \bar{\Upsilon}\left(x^{i}, t\right), \bar{\Upsilon}\left(x^{i}, t\right)\right]$ can be considered also in GR in order to construct inflation scenarios and dark energy and dark matter models.

\subsection{Parameterizations for cosmological d-metrics}

In this subsection, there are provided necessary coefficient formulas and examples of N-adapted calculus used in locally anisotropic cosmology with STQC structure. We consider certain basic representations for quadratic line elements describing nonholonomic deformations of prime metrics into target cosmological ones.

\subsubsection{Target d-metrics with polarization functions}

Let us consider a prime metric $\stackrel{\circ}{\mathrm{g}}=\stackrel{\circ}{g}_{\alpha \beta}\left(x^{i}, y^{a}\right) d u^{\alpha} \otimes d u^{\beta}$ which can be written in a N-adapted form

$$
\begin{aligned}
& \stackrel{\circ}{\mathrm{g}}=\stackrel{\circ}{g}_{\alpha}(u) \stackrel{\circ}{\mathbf{e}}^{\alpha} \otimes \stackrel{\circ}{ }^{\beta}=\stackrel{\circ}{g}_{i}(x, y) d x^{i} \otimes d x^{i}+\stackrel{\circ}{h}_{a}(x, y) \stackrel{\circ}{ }^{a} \otimes \stackrel{\circ}{ }^{a}, \text { for } \\
& \stackrel{\circ}{\mathbf{e}}^{\alpha}=\left(d x^{i}, \mathbf{e}^{a}=d y^{a}+\stackrel{\circ}{N}_{i}^{a}(u) d x^{i}\right) \text {, and } \stackrel{\circ}{\mathbf{e}}_{\alpha}=\left(\stackrel{\circ}{\mathbf{e}}_{i}=\partial / \partial y^{a}-\stackrel{\circ}{N}_{i}^{b}(u) \partial / \partial y^{b}, e_{a}=\partial / \partial y^{a}\right) \text {. }
\end{aligned}
$$

In general, such a d-metric can be, or not, a solution of some gravitational field equations in a MGT or GR. We can take $\stackrel{\mathrm{g}}{\text { (24) }}$ (2s a necessary type coordinate transform of a cosmological metric, for instance, of a Friedman-Lemaitre-Robertson-Walker (FLRW), or a Bianch type, metric. For a diagonalizable FLRW metric, we can always find a frame/coordinate system when $N_{i}^{b}=0$. It is convenient to work with local coordinate systems and nonzero values for $N_{i}^{b}$ which do not result in certain singular/pecular nonholonomic deformations as we shall describe below.

To generate new classes of cosmological solutions we shall consider nonholonomic deformations $\stackrel{\circ}{\mathrm{g}} \rightarrow \widehat{\mathrm{g}}=\left[g_{\alpha}=\eta_{\alpha} \stackrel{\circ}{\alpha}_{\alpha}, N_{i}^{a}=\eta_{i}^{a} \stackrel{\circ}{N}_{i}^{a}\right]$ with so-called $\eta$-polarization functions of a 'prime' metric, $\stackrel{\circ}{\mathrm{g}}$, into a 'target' metric $\mathbf{g}=\widehat{\mathbf{g}}$. In $\mathrm{N}$-adapted form, target d-metrics of type (3) are parameterized

$$
\begin{aligned}
\stackrel{\circ}{\mathrm{g}} & \rightarrow \widehat{\mathrm{g}}=g_{i}\left(x^{k}\right) d x^{i} \otimes d x^{i}+h_{a}\left(x^{k}, t\right) \mathbf{e}^{a} \otimes \mathbf{e}^{a} \\
& =\eta_{i}\left(x^{k}, y^{b}\right) \stackrel{\circ}{g}_{i} d x^{i} \otimes d x^{i}+\eta_{a}\left(x^{k}, y^{b}\right) \stackrel{\circ}{h_{a}} \mathbf{e}^{a}[\eta] \otimes \mathbf{e}^{a}[\eta],
\end{aligned}
$$

where the N-elongated basis (2) is written for $N_{i}^{a}(u)=\eta_{i}^{a}\left(x^{k}, y^{b}\right) \stackrel{\circ}{i}_{i}^{a}\left(x^{k}, y^{b}\right)$, i.e. in the form $\mathbf{e}^{\alpha}[\eta]=$ $\left(d x^{i}, \mathbf{e}^{a}=d y^{a}+\eta_{i}^{a} \stackrel{\circ}{i}_{i}^{a} d x^{i}\right)$. We shall subject a $\widehat{\mathbf{g}}$ to the condition that it defines a generalized cosmological solution of (modified) Einstein equations when coefficients of a corresponding d-metric and (non) linear connection depend at least on a time like coordinate $y^{4}=t$.

\footnotetext{
${ }^{6}$ In principle, we can generate exact solutions for parameterizations of metrics and sources depending on all spacetime coordinates but formulas for such solution are much more cumbersome. For our purposes, it is enough to consider effective source configurations of type (23).

${ }^{7}$ we do not consider summation on repeating indices if they are not written as contraction of "up-low" ones
} 
Target quadratic line elements can be represented in generic off-diagonal form, $\mathbf{g}_{\alpha \beta}=\left[g_{i}, h_{a}, n_{i}, w_{i}\right]$, and/or using $\eta$-polarization functions,

$$
\begin{aligned}
d s^{2}= & g_{i}\left(x^{k}\right)\left[d x^{i}\right]^{2}+h_{3}\left(x^{k}, t\right)\left[d y^{3}+n_{i}\left(x^{k}, t\right) d x^{i}\right]^{2}+h_{4}\left(x^{k}, t\right)\left[d t+w_{i}\left(x^{k}, t\right) d x^{i}\right]^{2} \\
= & \eta_{i}\left(x^{k}, t\right) \stackrel{\circ}{g}_{i}\left(x^{k}, t\right)\left[d x^{i}\right]^{2}+\eta_{3}\left(x^{k}, t\right) \stackrel{\circ}{h}_{3}\left(x^{k}, t\right)\left[d y^{3}+\eta_{i}^{3}\left(x^{k}, t\right) \stackrel{\circ}{N}_{i}^{3}\left(x^{k}, t\right) d x^{i}\right]^{2} \\
& +\eta_{4}\left(x^{k}, t\right) \stackrel{\circ}{h}{ }_{4}\left(x^{k}, t\right)\left[d t+\eta_{i}^{4}\left(x^{k}, t\right) \stackrel{\circ}{N}_{i}^{4}\left(x^{k}, t\right) d x^{i}\right]^{2} \\
= & \eta_{i} \stackrel{\circ}{g}_{i}\left[d x^{i}\right]^{2}+\eta_{3} \stackrel{\circ}{h}_{3}\left[d y^{3}+\eta_{k}^{3} \stackrel{\circ}{k}_{k}^{3} d x^{k}\right]^{2}+\eta_{4} \stackrel{\circ}{h}_{4}\left[d t+\eta_{k}^{4} \stackrel{\circ}{*}_{k}^{4} d x^{k}\right]^{2} .
\end{aligned}
$$

Let us consider a coordinate transform to a new time like coordinate $y^{4}=t \rightarrow \tau$ when $t=t\left(x^{i}, \tau\right)$,

$$
\begin{aligned}
d t= & \partial_{i} t d x^{i}+(\partial t / \partial \tau) d \tau ; d \tau=(\partial t / \partial \tau)^{-1}\left(d t-\partial_{i} t d x^{i}\right), \text { i.e. } \\
& (\partial t / \partial \tau) d \tau=\left(d t-\partial_{i} t d x^{i}\right) .
\end{aligned}
$$

We can rewrite the target d-metric using the new time variable $\tau$. For instance, the computations for the 4th term in (27) are

$$
\begin{aligned}
& \eta_{4} \stackrel{\circ}{h}_{4}\left[d t+\eta_{k}^{4} \stackrel{\circ}{N}_{k}^{4} d x^{k}\right]^{2}=\eta_{4} \stackrel{\circ}{h}_{4}\left[\partial_{k} t d x^{k}+(\partial t / \partial \tau) d \tau+\eta_{k}^{4} \stackrel{\circ}{N}_{k}^{4} d x^{k}\right]^{2} \\
& =\eta_{4}\left[\left(\partial_{k} t\right) d x^{k}+(\partial t / \partial \tau) d \tau+\eta_{k}^{4} \stackrel{\circ}{N}_{k}^{4} d x^{k}\right]^{2}=\eta_{4} \stackrel{\circ}{h}_{4}\left[(\partial t / \partial \tau) d \tau+\left(\partial_{k} t+\eta_{k}^{4} \stackrel{\circ}{N}_{k}^{4}\right) d x^{k}\right]^{2} \\
& =\stackrel{\circ}{h}_{4}\left[\eta_{4}(\partial t / \partial \tau) d \tau+\eta_{4}\left(\partial_{k} t+\eta_{k}^{4} \stackrel{\circ}{N}_{k}^{4}\right) d x^{k}\right]^{2}=\stackrel{\circ}{h}_{4}\left[\eta_{4}(\partial t / \partial \tau) d \tau+\eta_{4}\left(\partial_{k} t / \stackrel{\circ}{N}_{k}^{4}+\eta_{k}^{4}\right) \stackrel{\circ}{N}_{k}^{4} d x^{k}\right]^{2}
\end{aligned}
$$

Considering

$$
\begin{aligned}
\eta_{4}(\partial t / \partial \tau) & =1 ; \partial t / \partial \tau=\left(\eta_{4}\right)^{-1} \text { introduced for } d t=\partial_{i} t d x^{i}+(\partial t / \partial \tau) d \tau, \text { when } \\
d t & =\partial_{i} t d x^{i}+\left(\eta_{4}\right)^{-1} d \tau \text { for } \check{\eta}_{k}^{4}=\eta_{4}\left(\partial_{k} t / \stackrel{\circ}{N}_{k}^{4}+\eta_{k}^{4}\right) \stackrel{\circ}{N}_{k}^{4},
\end{aligned}
$$

a new time coordinate $\tau$ can be found from formulas $\partial t / \partial \tau=\left(\eta_{4}\right)^{-1}$ resulting in

$$
d \tau=\eta_{4}\left(x^{k}, t\right) d t ; \tau=\int \eta_{4}\left(x^{k}, t\right) d t+\tau_{0}\left(x^{k}\right) .
$$

Such coordinates with $\tau$ are useful for computations of nonholonomic deformations of the FLRW metrics.

\subsubsection{Parameterizations of prime d-metrics}

Let us consider a target line quadratic element for an off-diagonal cosmological solution in MGT written in the form (27). We can rewrite it using an effective target locally anisotropic cosmological scaling factor $\check{a}^{2}\left(x^{k}, \tau\right):=\eta\left(x^{k}, \tau\right) \grave{a}^{2}\left(x^{i}, \tau\right)$ with gravitational polarization $\eta\left(x^{k}, \tau\right)$ and prime cosmological scaling factor $\stackrel{\circ}{a}^{2}\left(x^{i}, \tau\right)$. This can be performed following formulas

$$
\begin{aligned}
& d s^{2}=\eta_{3}\left\{\frac{\eta_{i}}{\eta_{3}} \stackrel{\circ}{g}_{i}\left[d x^{i}\right]^{2}+\stackrel{\circ}{h}_{3}\left[d y^{3}+\eta_{k}^{3} \stackrel{\circ}{N}_{k}^{3} d x^{k}\right]^{2}\right\}+\stackrel{\circ}{h}_{4}\left[d \tau+\check{\eta}_{k}^{4} \stackrel{\circ}{N}_{k}^{4} d x^{k}\right]^{2} \\
& =\check{a}^{2}\left(x^{k}, \tau\right)\left\{\check{\eta}_{i}\left(x^{k}, \tau\right) \stackrel{\circ}{g}_{i}\left[d x^{i}\right]^{2}+\stackrel{\circ}{h}_{3}\left[d y^{3}+\check{\eta}_{k}^{3}\left(x^{k}, \tau\right) \stackrel{\circ}{N}_{k}^{3} d x^{k}\right]^{2}\right\}+\stackrel{\circ}{h}_{4}\left[d \tau+\check{\eta}_{k}^{4}\left(x^{k}, \tau\right) \stackrel{\circ}{N}_{k}^{4} d x^{k}\right]^{2} \\
& =\eta\left(x^{k}, \tau\right) \stackrel{\circ}{a}^{2}\left(x^{i}, \tau\right)\left\{\check{\eta}_{i}\left(x^{k}, \tau\right) \stackrel{\circ}{g}_{i}\left[d x^{i}\right]^{2}+\stackrel{\circ}{h}_{3}\left[d y^{3}+\check{\eta}_{k}^{3}\left(x^{k}, \tau\right) \stackrel{\circ}{N}_{k}^{3} d x^{k}\right]^{2}\right\}+\stackrel{\circ}{h}_{4}\left[d \tau+\check{\eta}_{k}^{4}\left(x^{k}, \tau\right) \stackrel{\circ}{N}_{k}^{4} d x^{k}\right]^{2} \text {, } \\
& \text { where } \check{a}^{2}\left(x^{k}, \tau\right):=\quad \eta_{3}\left(x^{k}, t\left(x^{i}, \tau\right)\right)=\eta\left(x^{k}, t\left(x^{i}, \tau\right)\right) \stackrel{a}{ }^{2}\left(x^{k}, t\left(x^{i}, \tau\right)\right)=\eta\left(x^{k}, \tau\right) \stackrel{a}{ }^{2}\left(x^{i}, \tau\right) \text {; } \\
& \check{\eta}_{i}\left(x^{k}, \tau\right):=\frac{\eta_{i}\left(x^{k}, t\left(x^{i}, \tau\right)\right)}{\eta\left(x^{k}, t\left(x^{i}, \tau\right)\right)} ; \check{\eta}_{k}^{3}\left(x^{k}, \tau\right):=\eta_{k}^{3}\left(x^{k}, t\left(x^{i}, \tau\right)\right) ; \\
& \check{\eta}_{k}^{4}\left(x^{k}, \tau\right):=\quad \eta_{4}\left\{\partial_{k} t\left(x^{i}, \tau\right)\left[\stackrel{\circ}{N}_{k}^{4}\left(x^{i}, t\left(x^{i}, \tau\right)\right)\right]^{-1}+\eta_{k}^{4}\left(x^{i}, t\left(x^{i}, \tau\right)\right)\right\} \stackrel{\circ}{N}_{k}^{4}\left(x^{i}, t\left(x^{i}, \tau\right)\right)
\end{aligned}
$$


If we consider a prime d-metric as a flat FLRW metric written in local coordinates

$$
\bar{u}=\left\{\bar{u}^{\alpha}\left(x^{i}, y^{3}, \tau\right)=\left(\bar{x}^{1}\left(x^{i}, y^{3}, \tau\right), \bar{x}^{2}\left(x^{i}, y^{3}, \tau\right), \bar{y}^{3}\left(x^{i}, y^{3}, \tau\right), \bar{y}^{4}\left(x^{i}, y^{3}, \tau\right)\right)\right\},
$$

a d-metric (26) can be written in curved coordinate form $\stackrel{\circ}{a}^{2}(\bar{u})$, with $\bar{u}^{\alpha}$, and/or using a prime cosmological scaling factor $\stackrel{\circ}{a}^{2}(\tau)$,

$$
\begin{aligned}
d \stackrel{\circ}{s}^{2} & =\stackrel{\circ}{a}^{2}(\bar{u})\left\{\stackrel{\circ}{g}_{i}(\bar{u})\left[d \bar{x}^{i}\right]^{2}+\stackrel{\circ}{h}_{3}(\bar{u})\left[d \bar{y}^{3}+\stackrel{\circ}{N}_{k}^{3}(\bar{u}) d \bar{x}^{k}\right]^{2}\right\}+\stackrel{\circ}{h}_{4}(\bar{u})\left[d \bar{y}^{4}+\stackrel{\circ}{N}_{k}^{4}(\bar{u}) d \bar{x}^{k}\right]^{2} \\
& \rightarrow \stackrel{\circ}{a}^{2}(\tau)\left[d x^{i}\right]^{2}-d \tau^{2}, \\
\text { for } \bar{u}^{\alpha} & \rightarrow\left(x^{i}, y^{3}, \tau\right), \stackrel{\circ}{g}_{i} \rightarrow 1, \stackrel{\circ}{h}_{3} \rightarrow 1, \stackrel{\circ}{h}_{4} \rightarrow-1, \stackrel{\circ}{N}_{k}^{a}(\bar{u}) \rightarrow 0 \text { and } \stackrel{\circ}{a}^{2}(\bar{u}) \rightarrow \stackrel{\circ}{a}^{2}(\tau) .
\end{aligned}
$$

Quasi FLRW configurations are determined (by definition) by a diagonalized solution in MGT or GR for the d-metric is of type (28) when the integration functions and coordinates result in $\check{\eta}_{k}^{a}\left(x^{k}, \tau\right)=0$,

$$
d s^{2}=\eta\left(x^{k}, \tau\right) \stackrel{\circ}{ }^{2}\left\{\check{\eta}_{i}\left(x^{k}, \tau\right) \stackrel{\circ}{g}_{i}\left[d x^{i}\right]^{2}+\stackrel{\circ}{h}_{3}\left[d y^{3}\right]^{2}\right\}+\stackrel{\circ}{h}_{4}[d \tau]^{2} .
$$

For partial small nonholonomic deformations, such d-metrics admit parameterizations $\check{\eta}_{i} \simeq 1+$ $\varepsilon \check{\chi}_{i}\left(x^{k}, \tau\right)$ when the polarization of the target cosmological factor, $\eta\left(x^{k}, \tau\right)$ can be a general one and not a value of type $1+\varepsilon \chi\left(x^{k}, \tau\right)$ with a small parameter $\varepsilon$. A resulting scaling factor $a^{2}\left(x^{k}, \tau\right)=$ $\eta\left(x^{k}, \tau\right) \grave{a}^{2}\left(x^{k}, \tau\right)$, with possible further re-parametrization or a limit to $a^{2}(\tau)=\eta(\tau) \dot{a}^{2}(\tau)$, encodes possible nonlinear off-diagonal and parametric interactions determined by systems of nonlinear PDEs.

\subsubsection{Approximations for target d-metrics}

To study properties of MGTs and cosmological models is convenient to consider different types of approximations for nonholonomic deformations of (29) to a target d-metric (28). Let us analyze six classes of exact, or parametric, solutions which can be generated by a respective subclass of generating functions and/or generating sources, or some diagonal approximations, or by introducing small $\varepsilon$-parameters.

1. We can chose mutual reparameterizations of generating functions $(\Psi, \Upsilon) \Longleftrightarrow(\Phi, \Lambda=$ const $)$ and integrating functions when the coefficients of a target d-metric $\widehat{\mathbf{g}}_{\alpha \beta}(\tau)$ depend only a time like coordinate $\tau$, when $\eta\left(x^{k}, \tau\right) \rightarrow \widetilde{\eta}(\tau)$ and $a\left(x^{k}, \tau\right) \rightarrow \widetilde{a}^{2}(\tau)=\widetilde{\eta}(\tau) \stackrel{\circ}{ }^{2}(\tau)$. Respective linear quadratic elements (28) can be represented in the form

$$
d s^{2}=\eta(\tau) \stackrel{\circ}{a}^{2}(\tau)\left\{\check{\eta}_{i}(\tau) \stackrel{\circ}{g}_{i}\left[d x^{i}\right]^{2}+\stackrel{\circ}{h}_{3}\left[d y^{3}+\check{\eta}_{k}^{3}(\tau) \stackrel{\circ}{N}_{k}^{3} d x^{k}\right]^{2}\right\}+\stackrel{\circ}{h}_{4}\left[d \tau+\check{\eta}_{k}^{4}(\tau) \stackrel{\circ}{N}_{k}^{4} d x^{k}\right]^{2} .
$$

With respect to coordinate bases, such cosmological solutions can be generic off-diagonal and could be chosen in some forms describing certain nonholonomic deformations of Bianchi cosmological models.

2. For FLRW prime configurations, we can consider generation functions and integration functions which result in zero values of the target N-connection coefficients (45) and/or consider limits $N_{k}^{a} \rightarrow 0$. For such cases, we transform (31) into diagonal metrics

$$
d s^{2}=\eta(\tau) \stackrel{\circ}{a}^{2}(\tau)\left\{\check{\eta}_{i}(\tau) \stackrel{\circ}{g}_{i}\left[d x^{i}\right]^{2}+\stackrel{\circ}{h}_{3}\left(d y^{3}\right)^{2}\right\}+\stackrel{\circ}{h}_{4}(d \tau)^{2}
$$

modeling certain locally anisotropic interactions with a "memory" of nonholonomic and offdiagonal structures. 
3. Small parametric nonholonomic deformations of a prime metric (29) into target off-diagonal cosmological solutions (28) can be described by approximations

$$
\check{\eta}_{i}\left(x^{k}, \tau\right) \simeq 1+\varepsilon_{i} \check{\chi}_{i}\left(x^{k}, \tau\right), \eta\left(x^{k}, \tau\right) \simeq 1+\varepsilon_{3} \chi\left(x^{k}, \tau\right), \check{\eta}_{k}^{a}\left(x^{k}, \tau\right) \simeq 1+\varepsilon_{k}^{a} \check{\chi}_{k}^{a}\left(x^{k}, \tau\right),
$$

where small parameters $\varepsilon_{i}, \varepsilon_{3}, \varepsilon_{k}^{a}$ satisfy conditions of type $0 \leq\left|\varepsilon_{i}\right|,\left|\varepsilon_{3}\right|,\left|\varepsilon_{k}^{a}\right| \ll 1$ and, for instance, $\chi\left(x^{k}, \tau\right)$ is taken as a generating function. Such approximations may impose certain relations between such $\varepsilon$-constants and $\chi$-functions and restrict the classe of generating functions subjected to nonlinear symmetries. Corresponding quadratic line elements are written

$$
\begin{aligned}
d s^{2}= & {\left[1+\varepsilon_{3} \chi\left(x^{k}, \tau\right)\right] \stackrel{\circ}{a}^{2}\left(x^{i}, \tau\right)\left\{\left[1+\varepsilon_{i} \check{\chi}_{i}\left(x^{k}, \tau\right)\right] \stackrel{\circ}{g}_{i}\left[d x^{i}\right]^{2}+\right.} \\
& \left.\stackrel{\circ}{h}_{3}\left[d y^{3}+\left(1+\varepsilon_{k}^{3} \check{\chi}_{k}^{3}\left(x^{k}, \tau\right)\right) \stackrel{\circ}{N}_{k}^{3} d x^{k}\right]^{2}\right\}+\check{\circ}_{4}\left[d \tau+\left(1+\varepsilon_{k}^{4} \check{\chi}_{k}^{4}\left(x^{k}, \tau\right)\right) \stackrel{\circ}{N}_{k}^{4} d x^{k}\right]^{2} .
\end{aligned}
$$

Such off-diagonal solutions define cosmological metrics with certain small independent fluctuations, for instance, a FLRW embedded self-consistently into a locally anisotropic

4. We can construct off-diagonal cosmological solutions with small parameters $\varepsilon_{i}, \varepsilon_{3}, \varepsilon_{k}^{a}$ when the generating functions and d-metric and N-connection coefficients do not depend on space like coordinates. For such approximations, the quadratic line element (33) transforms into

$$
\begin{aligned}
d s^{2}= & {\left[1+\varepsilon_{3} \chi(\tau)\right] \stackrel{\circ}{a}^{2}(\tau)\left\{\left[1+\varepsilon_{i} \check{\chi}_{i}(\tau)\right] \stackrel{\circ}{g}_{i}\left[d x^{i}\right]^{2}+\right.} \\
& \left.\stackrel{\circ}{h}\left[d y^{3}+\left(1+\varepsilon_{k}^{3} \check{\check{\chi}}_{k}^{3}(\tau)\right) \stackrel{\circ}{N}_{k}^{3} d x^{k}\right]^{2}\right\}+\stackrel{\circ}{h}_{4}\left[d \tau+\left(1+\varepsilon_{k}^{4} \check{\chi}_{k}^{4}(\tau)\right) \stackrel{\circ}{N}_{k}^{4} d x^{k}\right]^{2} .
\end{aligned}
$$

5. Off-diagonal deformations, for instance, of a FLRW metric into locally anisotropic cosmological solutions in a MGT or GR can be constructed using only one small parameter $\varepsilon=\varepsilon_{i}=\varepsilon_{3}=\varepsilon_{k}^{a}$, when (34) transforms into

$$
\begin{aligned}
d s^{2}= & {\left[1+\varepsilon \chi\left(x^{k}, \tau\right)\right] \stackrel{\circ}{a}^{2}\left(x^{i}, \tau\right)\left\{\left[1+\varepsilon \check{\chi}_{i}\left(x^{k}, \tau\right)\right] \stackrel{\circ}{g}_{i}\left[d x^{i}\right]^{2}+\right.} \\
& \left.\stackrel{\circ}{h}\left[d y^{3}+\left(1+\varepsilon \check{\chi}_{k}^{3}\left(x^{k}, \tau\right)\right) \stackrel{\circ}{N}_{k}^{3} d x^{k}\right]^{2}\right\}+\stackrel{\circ}{h}_{4}\left[d \tau+\left(1+\varepsilon \check{\chi}_{k}^{4}\left(x^{k}, \tau\right)\right) \stackrel{\circ}{N}_{k}^{4} d x^{k}\right]^{2} .
\end{aligned}
$$

6. Finally, we can impose on (35) the condition that the $\varepsilon$-deformations depend on a time like coordinate. This results in d-metrics

$$
\begin{aligned}
d s^{2}= & {[1+\varepsilon \chi(\tau)] \stackrel{\circ}{a}^{2}(\tau)\left\{\left[1+\varepsilon \check{\chi}_{i}(\tau)\right] \stackrel{\circ}{g}_{i}\left[d x^{i}\right]^{2}+\right.} \\
& \left.\stackrel{\circ}{h}_{3}\left[d y^{3}+\left(1+\varepsilon \check{\chi}_{k}^{3}(\tau)\right) \stackrel{\circ}{N}_{k}^{3} d x^{k}\right]^{2}\right\}+\stackrel{\circ}{h}_{4}\left[d \tau+\left(1+\varepsilon \check{\chi}_{k}^{4}(\tau)\right) \stackrel{\circ}{N}_{k}^{4} d x^{k}\right]^{2}
\end{aligned}
$$

which can be considered as some ansatz used, for instance, for describing quantum fluctuations of FLRW metrics in various MGTs.

Various classes of cosmological solutions with parametric $\varepsilon$-decompositions can be performed in a self-consistent form by omitting quadratic and higher terms after a class of solutions have been found for some general data $\left(\eta_{\alpha}, \eta_{i}^{a}\right)$. They are more general than approximate solutions found, for instance, for classical and quantum fluctuations of standard FLRW metrics. For certain subclasses of generic off-diagonal solutions, we can consider that $\varepsilon_{i}, \varepsilon_{a}, \varepsilon_{i}^{a} \sim \varepsilon$, when only one small parameter is considered for all coefficients of nonholonomic deformations. This way, we can generate various classes of nonholonomic small deformations of cosmological solutions like in Refs. [1, 2, 3, 4, 5, 6], see also references in those works citing constructions on locally anisotropic black hole/ wormhole and other type solutions. 


\section{Off-diagonal and quasi FLRW solutions with STQC struc- ture}

In this section, we show how the system of (modified) Einstein equations can be integrated in general off-diagonal forms for locally anisotropic and inhomogeneous cosmological solutions with one-Killing symmetry and parameterized by metrics of type $\overline{\mathbf{g}}_{\alpha \beta}\left[x^{i}, y^{a} ; \bar{\varsigma}, \bar{\chi}, \bar{q}, \bar{\psi}, \bar{q}, \ldots\right]$ (13). It is proven that after certain classes of such generalized cosmological metrics and connections in MGTs or GR have been found using geometric methods for integrating nonlinear PDEs we can always extract quasi FLRW configurations encoding nonlinear off-diagonal gravitational and matter field interactions. We emphasize that cosmological STQC-solutions can not be constructed if we work from the very beginning only with time depending metrics of type $\mathbf{g}_{\alpha \beta}(t)$. Such particular type time like parameterizations, or approximations, resulting in STQC-configurations must be considered at the end, when generalized solutions with dependencies both on time and space coordinates have been constructed. Such new classes of cosmological solutions will be applied in next sections for elaborating new cosmological scenarios with pattern forming and evolution of STQC-structures.

\subsection{Cosmological ansatz for STQC structures}

Any target d-metric (25) can be represented in a form (3) and/or in a quasi FLRW form [see details related to formula (30)], with Killing symmetry on $\partial / \partial y^{3}$,

$$
\begin{aligned}
d s^{2}= & g_{i}\left(x^{k}\right)\left[d x^{i}\right]^{2}+h_{3}\left(x^{k}, t\right)\left[d y^{3}+n_{i}\left(x^{k}, t\right) d x^{i}\right]^{2}+h_{4}\left(x^{k}, t\right)\left[d t+w_{i}\left(x^{k}, t\right) d x^{i}\right]^{2} \\
= & \eta_{i} \stackrel{\circ}{i}_{i}\left[\bar{e}^{i}\right]^{2}+\eta_{3} \stackrel{\circ}{h}_{3}\left[\overline{\mathbf{e}}^{3}\right]^{2}+\stackrel{\circ}{h}_{4}\left[\overline{\mathbf{e}}^{4}\right]^{2} \\
= & a^{2}\left(x^{k}, \tau\right)\left\{\check{\eta}_{i}\left(x^{k}, \tau\right) \stackrel{\circ}{g}_{i}\left[\check{e}^{i}\right]^{2}+\check{\circ}_{3}\left[\check{\mathbf{e}}^{3}\right]^{2}\right\}+\stackrel{\circ}{h}_{4}\left(\check{\mathbf{e}}^{4}\right)^{2}, \\
\text { where } \quad & \bar{e}^{i}=d x^{i}, \overline{\mathbf{e}}^{3}=d y^{3}+\eta_{k}^{3} \stackrel{\circ}{N}_{k}^{3} d x^{k}, \overline{\mathbf{e}}^{4}=\sqrt{\left|\eta_{4}\right|}\left(d t+\eta_{k}^{4} \stackrel{\circ}{k}_{k}^{4} d x^{k}\right), \\
& \check{e}^{i}=d x^{i}, \check{\mathbf{e}}^{3}=d y^{3}+\check{\eta}_{k}^{3}\left(x^{k}, \tau\right) \stackrel{\circ}{N}_{k}^{3} d x^{k}, \check{\mathbf{e}}^{4}=d \tau+\check{\eta}_{k}^{4}\left(x^{k}, \tau\right) N_{k}^{4} d x^{k} .
\end{aligned}
$$

The formulas for frame and d-metric coefficient transforms in (37), (38) and (39), and for certain parametric decompositions and approximations, see (27) and (28), and (31)-(36). Here we note that the coordinate transforms $u^{\alpha}=\left(x^{i}, y^{3}, y^{4}=t\left(x^{i}, \tau\right)\right) \rightarrow\left(x^{i}, y^{3}, \tau\right)$ are subjected to the conditions $d \tau=\eta_{4}\left(x^{k}, t\right) d t$ and

$$
\begin{aligned}
\sqrt{\left|h_{3}\right|} & =\sqrt{\left|\eta_{3} \stackrel{\circ}{h}\right|}=a\left(x^{i}, t\left(x^{i}, \tau\right)\right)=\check{a}\left(x^{i}, \tau\right)=\sqrt{\left|\eta\left(x^{i}, \tau\right)\right|} \stackrel{\circ}{a}\left(x^{i}, \tau\right), \\
& \simeq a^{2}\left(x^{k}, \tau\right) \rightarrow \widetilde{a}^{2}(\tau)=\widetilde{\eta}(\tau) \stackrel{\circ}{ }^{2}(\tau) \text { for nonholonomic generation of FLRW configurations. }
\end{aligned}
$$

These formulas can be considered for $\eta_{3}=\eta\left(x^{i}, t\right)$ and $h_{3}=\eta_{3} \grave{h}_{3}=a^{2}=\eta \grave{a}^{2}$. If we choose parameterizations and limits of solutinos with $\check{\eta}_{i}(\tau)=\eta(\tau)=1, \stackrel{\circ}{g}_{i}=\stackrel{\circ}{h}_{3}=1$ and $\stackrel{\circ}{h}_{4}=-1$, see (29), we obtain the flat FLRW metric used for elaborating locally isotropic and homogeneous theories. For some nontrivial values, the polarization functions $\eta\left(x^{i}, \tau\right)$ or $\tilde{\eta}(\tau)$ define respective nonholonomic deformations of some primary data $\stackrel{\circ}{a}\left(x^{i}, t\right)$ or $\stackrel{\circ}{a}(t)$. Such $\eta$-functions can be found by constructing generic off-diagonal solutions of gravitational field equations in a MGT or GR.

\subsection{Systems of nonlinear PDEs for cosmological STQCs}

Off-diagonal cosmological solutions can be found in explicit form for ansatz (37) if we apply the anholonomic frame deformation method, AFDM, see details in Appendix A.2.2 of ref. [6] (here we 
note that we use a different system of notation in this work). Computing respective Ricci d-tensors, the nonholonomic deformations of the Einstein equations (18) for the canonical d-connection $\widehat{\mathbf{D}}$ and sources (22) can be written as a system of nonlinear PDEs,

$$
\begin{aligned}
\left(\partial_{11}^{2}+\partial_{22}^{2}\right) \ln \left|g_{1}\right| & =2{ }_{h} \Upsilon \\
(\ln |\Psi|)^{\bullet} h_{3}^{\bullet} & =2 h_{3} h_{4} \Upsilon \\
n_{i}^{\bullet \bullet}+\gamma n_{i}^{\bullet} & =0, \\
\beta w_{i}-\alpha_{i} & =0 .
\end{aligned}
$$

The coefficients in these formulas are defined

$$
\begin{gathered}
\alpha_{i}=h_{3}^{\bullet} \partial_{i} \ln |\Psi|, \beta=h_{3}^{\bullet}(\ln |\Psi|)^{\bullet}, \gamma=\left(\ln \left|h_{3}\right|^{3 / 2} / h_{4}\right)^{\bullet} \\
\text { where } \Psi\left(x^{i}, t\right)=2\left(\sqrt{\left|h_{3}\right|}\right)^{\bullet} / \sqrt{\left|h_{4}\right|}
\end{gathered}
$$

for $h_{a}^{\bullet} \neq 0$ and $(\ln |\Psi|)^{\bullet} \neq 0$ and $g_{1}\left(x^{k}\right)=g_{2}\left(x^{k}\right)$. We use such systems of references and coordinates when these conditions are satisfied.

The system (41) can integrated for any generating function $h_{4}$ and sources ${ }_{h} \Upsilon\left(x^{i}\right)$ and $\Upsilon\left(x^{k}, t\right)$. Alternatively, we can take $\Psi\left(x^{i}, t\right)$ as a generating function and then find the coefficients of the v-metric and N-connection.

\subsection{Generating functions and integrals for off-diagonal cosmological solu- tions}

The system (41) and (42) possess a property of decoupling for unknown variables $g_{1}, h_{a}, n_{i}$ and $w_{i}$, for a generating function $\Psi$, see similar details in [1, 2, 3, 6]. Integrating consequently, we generate exact solutions for the modified Einstein equations (18). Here we note an important nonlinear symmetry following from the second lines in (41) and (42). Such equations can be written for the cosmological scaling factor $a$,

$$
\begin{aligned}
(\ln |\Psi|)^{\bullet} a^{\bullet} & =a h_{4} \Upsilon \\
\Psi\left(x^{i}, t\right) & =2 a^{\bullet} / \sqrt{\left|h_{4}\right|}
\end{aligned}
$$

which allows us to redefine the generating functions and generating sources, $(\Psi, \Upsilon) \Longleftrightarrow(\Phi, \Lambda=$ const), subjected to the conditions

$$
\frac{\left(\Psi^{2}\right)^{\bullet}}{|\Upsilon|}=\frac{\left(\Phi^{2}\right)^{\bullet}}{\Lambda}, \text { or } \Lambda \Psi^{2}=\Phi^{2}|\Upsilon|-\int d t \Phi^{2}|\Upsilon|^{\bullet}
$$

Using such formulas, we introduce a new generating function $\Phi\left(x^{i}, t\right)$ and an (effective) cosmological constant $\Lambda \neq 0$ (for zero values, there are necessary more special considerations), which can be applied for generating exact off-diagonal solutions in explicit forms. The value $\Lambda$ is fixed by observational data (for instance, for the dark energy encoding contributions of nonlinear gravitational interactions with nontrivial vacuum) or computed from classical/quantum models. We can consider models with splitting of the cosmological constant into contributions from modified gravity, matter fields and effective sources from STQC structures, $\Lambda={ }^{F} \Lambda+{ }^{m} \Lambda+\bar{\Lambda}$. 
In $\mathrm{N}$-adapted and/or equivalent off-diagonal forms, generic off-diagonal solutions with Killing symmetry on $\partial / \partial y^{3}$ are determined by such coefficients of $\mathrm{d}-$-metrics,

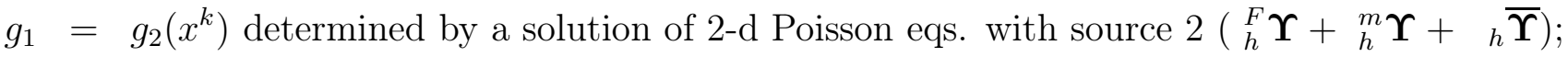

$$
\begin{aligned}
& h_{3}=a^{2}=h_{3}^{[0]}\left(x^{k}\right)-\int d t \frac{\left(\Psi^{2}\right)^{\bullet}}{4 \Upsilon}=h_{3}[\Phi]=h_{3}^{[0]}\left(x^{k}\right)-\frac{\Phi^{2}}{4 \Lambda}
\end{aligned}
$$

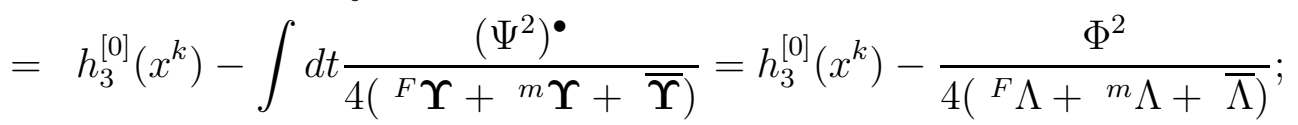

$$
\begin{aligned}
& h_{4}=-\frac{\left(\Psi^{2}\right)^{\bullet}}{4 \Upsilon^{2} h_{3}}=-\frac{\left(\Psi^{2}\right)^{\bullet}}{4\left({ }^{F} \Upsilon+{ }^{m} \Upsilon+\bar{\Upsilon}\right)^{2} h_{3}} \\
& =-\frac{\left|\left(\Phi^{2}\right)^{\bullet}\right|^{2}}{4 h_{3}[\Phi]\left|\left({ }^{F} \Lambda+{ }^{m} \Lambda+\bar{\Lambda}\right) \int d t\left({ }^{F} \Upsilon+{ }^{m} \boldsymbol{\Upsilon}+\bar{\Upsilon}\right)\left(\Phi^{2}\right)^{\bullet}\right|^{\prime}} ;
\end{aligned}
$$

and the coefficients of $\mathrm{N}$-connection,

$$
\begin{aligned}
N_{k}^{3} & =n_{k}\left(x^{i}, t\right)={ }_{1} n_{k}\left(x^{i}\right)+{ }_{2} n_{k}\left(x^{i}\right) \int d t \frac{\left(\Phi^{\bullet}\right)^{2}}{4\left|\Lambda \int d t \boldsymbol{\Upsilon}\left(\Phi^{2}\right)^{\bullet}\right|\left|h_{3}\right|^{5 / 2}} \\
& ={ }_{1} n_{k}\left(x^{i}\right)+{ }_{2} n_{k}\left(x^{i}\right) \int d t \frac{\left(\Phi^{\bullet}\right)^{2}}{4\left|\left({ }^{F} \Lambda+{ }^{m} \Lambda+\bar{\Lambda}\right)\left(F \boldsymbol{T}+{ }^{m} \boldsymbol{\Upsilon}+\overline{\mathbf{\Upsilon}}\right)\left(\Phi^{2}\right)^{\bullet}\right|\left|h_{3}\right|^{5 / 2}} \\
N_{i}^{4} & =w_{i}\left(x^{i}, t\right)=\frac{\partial_{i} \Psi}{\Psi^{\bullet}}=\frac{\partial_{i}\left[\int d t \Upsilon\left(\Phi^{2}\right)^{\bullet}\right]}{\Upsilon\left(\Phi^{2}\right)^{\bullet}}=\frac{\partial_{i}\left[\int d t\left({ }^{F} \boldsymbol{\Upsilon}+{ }^{m} \boldsymbol{\Upsilon}+\overline{\mathbf{\Upsilon}}\right)\left(\Phi^{2}\right)^{\bullet}\right]}{\left({ }^{F} \boldsymbol{\Upsilon}+{ }^{m} \boldsymbol{\Upsilon}+\overline{\mathbf{\Upsilon}}\right)\left(\Phi^{2}\right)^{\bullet}}
\end{aligned}
$$

In above formulas, the values $h_{3}^{[0]}\left(x^{k}\right),{ }_{1} n_{k}\left(x^{i}\right)$, and ${ }_{2} n_{k}\left(x^{i}\right)$ are integration functions encoding various possible sets of (non) commutative parameters and integration constants.

Any coefficient $h_{3}$ with $h_{3}^{\bullet} \neq 0$, in (44) can be considered also as a generating function. Using (43)), we express the generating functions in terms of

$$
\begin{aligned}
\left(\Psi^{2}\right)^{\bullet} & =-\int d t \Upsilon h_{3}^{\bullet}=-\int d t\left({ }^{F} \Upsilon+{ }^{m} \Upsilon+\bar{\Upsilon}\right) h_{3}^{\bullet} \\
\Phi^{2} & =-4 \Lambda h_{3}=-4\left({ }^{F} \Lambda+{ }^{m} \Lambda+\bar{\Lambda}\right) h_{3} .
\end{aligned}
$$

All formulas (44), (45) and (46) can be rewirtten in terms of $\eta$-polarization functions d-metrics (25). This allows us to compute in explicit form the d-metric and N-connection coefficients, and respective nonlinear symmetries, for nonholonomic deformations of certain given prime d-metrics into new classes of generic off-diagonal, or diagonalized, cosmological solutions with "memory" on such deformations.

We can chose some generating data $(\Psi, \Upsilon)$, or $(\Phi, \Lambda)$, related by nonlinear differential/integral symmetries (43), and respective integration functions in explicit form following certain topology/ symmetry / asymptotic conditions for some classes of exact, or small parametric, cosmological solutions. The coefficients (44) define generic off-diagonal locally anisotropic cosmological solutions if the corresponding anholonomy coefficients $C_{\alpha \beta}^{\gamma}\left(x^{i}, t\right)$ (see footnote 2) are not trivial. Such solutions are with a nontrivial d-torsion induced nonholonomically $\mathrm{N}$-adapted coefficients which can be computed in explicit form. In order to generate as particular cases some well-known cosmological FLRW, or Bianchi, type metrics, we have to consider data of type $(\Psi(t), \Upsilon(t))$, or $(\Phi(t), \Lambda)$, with integration functions which allow frame/ coordinate transforms to respective (off-) diagonal configurations $g_{\alpha \beta}(t)$. 


\subsubsection{Extracting Levi-Civita STQC configurations}

Such configurations with $\widehat{\mathbf{D}}_{\mid \widehat{\mathcal{T}} \rightarrow 0}=\nabla$ can be defined by additional nonholonomic constraints (additional systems of PDEs on the coefficients of the canonical d-metric and N-connection) or certain limits, resulting in zero torsion $\widehat{\mathcal{T}}=0$. Such LC-conditions for (44) are satisfied if

$$
\begin{aligned}
\partial_{t} w_{i} & =\left(\partial_{i}-w_{i} \partial_{t}\right) \ln \sqrt{\left|h_{4}\right|},\left(\partial_{i}-w_{i} \partial_{t}\right) \ln \sqrt{\left|h_{3}\right|}=0, \\
\partial_{k} w_{i} & =\partial_{i} w_{k}, \partial_{t} n_{i}=0, \partial_{i} n_{k}=\partial_{k} n_{i} .
\end{aligned}
$$

This system of nonlinear first order PDEs can be solved in explicit form by imposing additional nonholonomic constraints on cosmological d-metrics and N-coefficients of (modified) Einstein equations. We can construct solutions of (47) in explicit form if the generation functions and generating sources in (44) and (45) are subjected to certain integrability conditions, for instance,

$$
\Psi=\check{\Psi}\left(x^{i}, t\right),\left(\partial_{i} \check{\Psi}\right)^{\bullet}=\partial_{i}\left(\check{\Psi}^{\bullet}\right) \text { and } \Upsilon\left(x^{i}, t\right)=\Upsilon[\check{\Psi}]=\check{\Upsilon} \text {, or } \Upsilon=\text { const },
$$

where $\Upsilon[\check{\Psi}]$ sates that possible v-sources depends via space-time coordinates only as functionals of $\check{\Psi}$.

Expressing

$$
\check{h}_{3}=h_{3}^{[0]}\left(x^{k}\right)-\int d t \frac{\left(\check{\Psi}^{2}\right)^{\bullet}}{4 \check{\Upsilon}}=h_{3}^{[0]}\left(x^{k}\right)-\frac{\check{\Phi}^{2}}{4 \Lambda},
$$

we can check by explicit computations that $\widehat{\mathcal{T}}=0$ if the N-connection coefficients are taken

$$
\begin{aligned}
& n_{k}=\check{n}_{k}=\partial_{k} n\left(x^{i}\right) \text { and }
\end{aligned}
$$

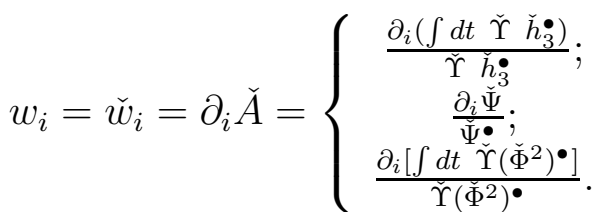

A function $\check{A}\left(x^{i}, t\right)$ can be prescribed in a necessary form which restricts the class of possible generating functions subjected, for instance, to the condition that it is a solution of the equation

$$
\left(\partial_{i} \check{A}\right) \check{\Psi} \bullet=\partial_{i} \check{\Psi} .
$$

Such solutions can be found in explicit or parametric forms if (inversely) we prescribe $\check{\Psi}$ but compute $\check{A}$ as a solution of the same equation.

For LC-configurations, d-metrics of type (37), (38) and (39) can be written respectively

$$
\begin{aligned}
d s^{2}= & g_{i}\left(x^{k}\right)\left[d x^{i}\right]^{2}+h_{3}\left(x^{k}, t\right)\left[d y^{3}+\partial_{k} n\left(x^{i}\right) d x^{k}\right]^{2}+h_{4}\left(x^{k}, t\right)\left[d t+\partial_{i} \check{A}\left(x^{k}, t\right) d x^{i}\right]^{2} \\
= & \eta_{i} \stackrel{\circ}{g}_{i}\left[\bar{e}^{i}\right]^{2}+\eta_{3} \grave{h}_{3}\left[\overline{\mathbf{e}}^{3}\right]^{2}+\check{h}_{4}\left[\check{\mathbf{e}}^{4}\right]^{2} \\
= & a^{2}\left(x^{k}, \tau\right)\left\{\check{\eta}_{i}\left(x^{k}, \tau\right) \stackrel{g}{g}_{i}\left[\check{e}^{i}\right]^{2}+\check{h}_{3}\left[\check{\mathbf{e}}^{3}\right]^{2}\right\}+\check{h}_{4}\left(\check{\mathbf{e}}^{4}\left(x^{k}, \tau\right)\right)^{2}, \\
\text { where } \quad & \bar{e}^{i}=d x^{i}, \overline{\mathbf{e}}^{3}=d y^{3}+\partial_{k} n\left(x^{i}\right) d x^{k}, \check{\mathbf{e}}^{4}=\sqrt{\left|\eta_{4}\right|}\left(d t+\partial_{i} \check{A}\left(x^{k}, t\right) d x^{k}\right), \\
& \check{e}^{i}=d x^{i}, \check{\mathbf{e}}^{3}=d y^{3}+\partial_{k} n\left(x^{i}\right) d x^{k}, \check{\mathbf{e}}^{4}\left(x^{k}, \tau\right)=d \tau+\partial_{i} \check{A}\left(x^{k}, \tau\right) d x^{k} .
\end{aligned}
$$

For simplicity, in this work we shall provide formulas for canonical d-connections considering that LC-configurations can be always extracted by imposing additional constraints on generating/intergation functions and sources as in formulas (48), (50) and (51). 


\subsubsection{Constructing d-metrics and N-connections from STQC generating functions}

Cosmological d-metrics $\overline{\mathbf{g}}_{\alpha \beta}$ (13) describing STQC configurations can be generated by nonlinear off-diagonal interactions of gravitational and matter fields. For our further considerations, it is convenient to work with $\eta$-polarization functions introduced for the d-metric (25). We shall overline such values and generated coefficients of d-metrics and N-connections in order to emphasize that they are generated for polarization functions constructed as functionals on solutions for STQC structures. For instance, we shall write

$$
\eta_{3} \rightarrow \bar{\eta}=\bar{\eta}_{3}\left(x^{i}, t\right)=\bar{\eta}_{3}[\bar{\zeta}, \bar{\chi}, \bar{q}, \bar{\psi}, \bar{q}, \ldots]
$$

depending on certain data $\bar{\varsigma}\left(x^{i}, y^{a}\right)$ (6), $\bar{\chi}\left(x^{i}, y^{a}\right)$ and $\bar{q}\left(x^{i}, y^{a}\right)$ from (99), $\bar{\psi}\left(x^{i}, y^{a}\right)$ (11); and/or $\bar{\chi}\left(x^{i}, y^{a}\right)$ and $\bar{q}\left(x^{i}, y^{a}\right)$ from see (12), defined by solutions of respective nonlinear dynamical and/or evolution equations. The formula (40) for STQC nonholonomic deformations in (25) is written in "overlined" form, $\bar{h}_{3}=\bar{a}^{2}=\bar{\eta}^{\circ}{ }^{2}$, where $\stackrel{\circ}{a}$ is a prime cosmological scaling factor taken, for instance, for a FLRW model.

The formulas for nonlinear symmetries of generating functions of STQC systems (46) can be written in terms of polarization function $\bar{\eta}_{4}$, generating (effective) matter sources, and respectively associated (effective) cosmological constants,

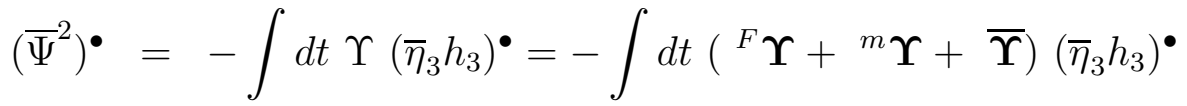

$$
\begin{aligned}
& \bar{\Phi}^{2}=-4 \Lambda\left(\bar{\eta}_{3} h_{3}\right)=-4\left({ }^{F} \Lambda+{ }^{m} \Lambda+\bar{\Lambda}\right)\left(\bar{\eta}_{3} h_{3}\right) .
\end{aligned}
$$

Such formulas allow us to rewrite, respectively, (44) and (45) in terms of $\bar{\eta}$-polarizations,

$$
\begin{aligned}
& \bar{\eta}_{i}\left(x^{k}, t\right)=\frac{g_{1}\left(x^{k}\right)}{g_{i}\left(x^{k}, t\right)} \text { with } g_{1}=g_{2}\left(x^{k}\right) \text { as a solution of 2-d Poisson eqs. with source } 2 \Upsilon \text {; } \\
& \bar{\eta}_{3}\left(x^{k}, t\right)=\bar{\eta} \text { can be considered as a generating function, or } \\
& \bar{\eta}=\stackrel{\circ}{h}_{3}^{-1}\left(h_{3}^{[0]}-\int d t \frac{\left(\bar{\Psi}^{2}\right)^{\bullet}}{4 \Upsilon}\right)=\stackrel{\circ}{h}_{3}^{-1}\left(h_{3}^{[0]}-\frac{\bar{\Phi}^{2}}{4 \Lambda}\right) ; \\
& \bar{\eta}_{4}\left(x^{k}, t\right)=\frac{4\left[\left(\left|\bar{\eta} h_{3}\right|^{1 / 2}\right)^{\bullet}\right]^{2}}{h_{4}\left|\int d t \Upsilon\left(\bar{\eta} h_{3}\right)^{\bullet}\right|} \\
& \bar{\eta}_{k}^{3}\left(x^{k}, t\right)=\frac{{ }_{1} n_{k}}{\grave{o}_{k}}+4 \frac{{ }_{2} n_{k}}{\stackrel{\circ}{n}_{k}} \int d t \frac{\left[\left(\left|\bar{\eta} h_{3}\right|^{-1 / 4}\right)^{\bullet}\right]^{2}}{\left|\int d t \Upsilon\left(\bar{\eta} h_{3}\right)^{\bullet}\right|} ; \bar{\eta}_{i}^{4}\left(x^{k}, t\right)=\frac{\partial_{i}\left[\int d t \Upsilon\left(\bar{\eta} h_{3}\right)^{\bullet}\right]}{\stackrel{\circ}{w}_{i} \Upsilon\left(\bar{\eta} h_{3}\right)^{\bullet}} .
\end{aligned}
$$

In these formulas, there are considered integration functions ${ }_{1} n_{k}\left(x^{k}\right),{ }_{2} n_{k}\left(x^{k}\right)$, and $h_{4}^{[0]}\left(x^{k}\right)$; and data for a primary cosmological metric $\stackrel{\mathrm{g}}{\mathrm{g}}$ (24), which can be prescribed, for instance, as a FLRW, or Bianchi type, d-metric.

Using formulas (40) and $\bar{\eta}$-polarizations (53), we can generate various classes of exact solutions of modified Einstein equations (18). Such d-metrics and related N-connections are parameterized in the form (25),

$$
\begin{gathered}
\stackrel{\circ}{\mathrm{g}} \rightarrow \overline{\mathbf{g}}=\bar{g}_{i}\left(x^{k}\right) d x^{i} \otimes d x^{i}+\bar{h}_{a}\left(x^{k}, t\right) \overline{\mathbf{e}}^{a} \otimes \overline{\mathbf{e}}^{a}=\bar{\eta}_{i} \stackrel{\circ}{g}_{i} d x^{i} \otimes d x^{i}+\bar{\eta}_{a} \stackrel{\circ}{h}_{a} \overline{\mathbf{e}}^{a}[\bar{\eta}] \otimes \overline{\mathbf{e}}^{a}[\bar{\eta}], \\
\text { where } \bar{N}_{i}^{a}=\bar{\eta}_{i}^{a} \stackrel{\circ}{N}_{i}^{a} \text { define } \overline{\mathbf{e}}^{a}[\bar{\eta}]=\left(d x^{i}, \mathbf{e}^{a}=d y^{a}+\bar{\eta}_{i}^{a} \stackrel{\circ}{N}_{i}^{a} d x^{i}\right) .
\end{gathered}
$$


The STQC character of such solutions is determined both by a gravitational generating function taken as a polarization $\bar{\eta}$; and a respective locally anisotropic cosmological scaling factor $\bar{a}^{2}=\bar{\eta} \stackrel{\circ}{ }^{2}$ (40) and by the components of a generating source for (effective) matter $\Upsilon={ }^{F} \Upsilon+{ }^{m} \Upsilon+\bar{\Upsilon}$. We emphasize that gravitational STQC configurations can be generated by usual matter fields, ${ }^{m} \Upsilon$, and effective sources in MGTs, ${ }^{F} \Upsilon$, even the STQC matter source is taken $\bar{\Upsilon}=0$.

We also note that generic off-diagonal nonlinear interactions may generate STQC configurations in GR for ${ }^{F} \Upsilon=0$. The LC-configurations are extracted as in previous subsection 3.3 .1 when, for instance, we have to solve the equation (51) rewritten for STQC generating functions, $\left(\partial_{i} \bar{\AA}\right) \overline{\check{\Psi}^{\bullet}}=\partial_{i} \overline{\check{\Psi}^{4}}$. This restricts the class of possible generating polarization functions $\bar{\eta}_{a} \rightarrow \bar{\eta}_{a}$ and generating sources $\check{\Upsilon}$. In general, cosmological STQC-configurations are described by generic off-diagonal metrics. Nevertheless, we can always prescribe/extract diagonal configurations as it is described by formulas for small $\varepsilon$-deformations (33)-(36). Quasi FLRW configurations can be generated also by a subclass of generating functions and sources $\check{\Psi}(t)$ and $\check{\Upsilon}(t)$. If we consider such prescriptions before constructing a general off-diagonal locally anisotropic cosmological solution, we restrict our considerations only to trivial nonholonomic deformations of diagonal cosmological metrics into another cosmological metrics $g_{\alpha}(t)$. In such cases, we lose the possibility to find cosmological solutions of (modified) Einstein equations describing certain STQC or QC structures. The key point is to apply the AFDM as we explained in details in [1, 2, 3, 6] and construct general off-diagonal d-metrics. At the end, we can consider $\varepsilon$-deformations and/or constraints to $\check{\Psi}(t)$ and $\check{\Upsilon}(t)$, with $h_{t}^{[0]}=$ const and ${ }_{1} n_{k}\left(x^{k}\right)=0$ and ${ }_{2} n_{k}\left(x^{k}\right)=0$, which would result in (diagonal) quasi FLRW metrics. This encodes STQC structures (with a memory/code for more general off-diagonal nonlinear interactions) via constants and parameters introduced for STQC dynamical and/or evolution equations, for instance, of type (6) , (99), (11); and/or (12).

\subsubsection{Off-diagonal quadratic line elements with nonholonomic STQC-torsion}

Putting together in (54) the STCS $\bar{\eta}$-polarization functions (53), we obtain general formulas for quadratic line elements determined by off-diagonal cosmological solutions with Killing symmetry on

$\partial_{3}$ determined by sources, ${ }_{h} \Upsilon={ }_{h}^{F} \Upsilon+{ }_{h}^{m} \Upsilon+{ }_{h} \bar{\Upsilon}$ and $\Upsilon={ }^{F} \Upsilon+{ }^{m} \Upsilon+\bar{\Upsilon}$, and an effective nontrivial cosmological constant $\Lambda={ }^{F} \Lambda+{ }^{m} \Lambda+\bar{\Lambda}$. In explicit form,

$$
\begin{aligned}
d s^{2} & =g_{1}\left(x^{k}\right)\left[\bar{e}^{i}\right]^{2}-\bar{\eta} \stackrel{\circ}{h}_{3}\left[\overline{\mathbf{e}}^{3}\right]^{2}+\stackrel{\circ}{h}_{4}\left[\overline{\mathbf{e}}^{4}\right]^{2} \\
& =\check{a}^{2}\left(x^{k}, \tau\right)\left\{\bar{\eta}_{i}\left(x^{k}, \tau\right) \stackrel{\circ}{g}_{i}\left[\check{e}^{i}\right]^{2}+\stackrel{\circ}{h}_{3}\left[\check{\mathbf{e}}^{3}\right]^{2}\right\}+\stackrel{\circ}{h}_{4}\left(\check{\mathbf{e}}^{4}\right)^{2},
\end{aligned}
$$

where

$$
\begin{aligned}
& \bar{e}^{i}=\check{e}^{i}=d x^{i} \\
& \overline{\mathbf{e}}^{3}=d y^{3}+\left(\frac{{ }_{1} n_{k}}{\stackrel{\circ}{n}_{k}}+4 \frac{{ }_{2} n_{k}}{\stackrel{\circ}{n}_{k}} \int d t \frac{\left[\left(\left|\bar{\eta} h_{3}\right|^{-1 / 4}\right)^{\bullet}\right]^{2}}{\left|\int d t\left(F \Upsilon+{ }^{m} \Upsilon+\bar{\Upsilon}\right)\left(\bar{\eta} h_{3}\right)^{\bullet}\right|}\right) \stackrel{\circ}{k}_{k}^{3} d x^{k}, \\
& \overline{\mathbf{e}}^{4}=\sqrt{\left|\bar{\eta}_{4}\right|}\left[d t+\frac{\partial_{i}\left[\int d t \Upsilon\left(\bar{\eta} h_{3}\right)^{\bullet}\right]}{\stackrel{\circ}{w}_{i} \Upsilon\left(\bar{\eta} h_{3}\right)^{\bullet}} \stackrel{\circ}{k}_{k}^{4} d x^{k}\right], \\
& \overline{\mathbf{e}}^{3}=d y^{3}+\bar{\eta}_{k}^{3}\left(x^{k}, \tau\right) \stackrel{\circ}{N}_{k}^{3} d x^{k}, \overline{\mathbf{e}}^{4}=d \tau+\bar{\eta}_{k}^{4}\left(x^{k}, \tau\right) \stackrel{\circ}{N}_{k}^{4} d x^{k} \text {. }
\end{aligned}
$$

The coefficients for polarization functions in (55) are defined and computed following formulas 
(28) but considering STCS $\bar{\eta}$-polarization functions (53), when

$$
\begin{aligned}
& \bar{\eta}_{3}\left(x^{k}, t\left(x^{i}, \tau\right)\right)=\bar{\eta}\left(x^{k}, t\left(x^{i}, \tau\right)\right), \text { see formulas (152); } \\
& \check{a}^{2}\left(x^{k}, \tau\right):=\quad \bar{\eta}_{3}\left(x^{k}, t\left(x^{i}, \tau\right)\right) \grave{a}^{2}\left(x^{k}, t\left(x^{i}, \tau\right)\right)=\bar{\eta}\left(x^{k}, t\left(x^{i}, \tau\right)\right) \grave{a}^{2}\left(x^{k}, t\left(x^{i}, \tau\right)\right)=\bar{\eta}\left(x^{k}, \tau\right) \grave{a}^{2}\left(x^{i}, \tau\right) ; \\
& \bar{\eta}_{i}\left(x^{k}, \tau\right):=\quad \frac{\bar{\eta}_{i}\left(x^{k}, t\left(x^{i}, \tau\right)\right)}{\bar{\eta}\left(x^{k}, t\left(x^{i}, \tau\right)\right)} ; \bar{\eta}_{k}^{3}\left(x^{k}, \tau\right):=\bar{\eta}_{k}^{3}\left(x^{k}, t\left(x^{i}, \tau\right)\right) ; \\
& \bar{\eta}_{k}^{4}\left(x^{k}, \tau\right):=\quad \bar{\eta}_{4}\left\{\partial_{k} t\left(x^{i}, \tau\right)\left[\stackrel{\circ}{N}_{k}^{4}\left(x^{i}, t\left(x^{i}, \tau\right)\right)\right]^{-1}+\bar{\eta}_{k}^{4}\left(x^{i}, t\left(x^{i}, \tau\right)\right)\right\} \stackrel{\circ}{N}_{k}^{4}\left(x^{i}, t\left(x^{i}, \tau\right)\right) .
\end{aligned}
$$

Such locally anisotropic and inhomogeneous cosmological solutions are, in general, with nontrivial nonholonomically induced torsion. This class of solutions can be re-defined equivalently in terms of generating functions $\bar{\Psi}\left(x^{k}, t\right)$ and/or $\bar{\Phi}\left(x^{k}, t\right)$, see $\bar{\eta}_{4}\left(x^{k}, t\right)=\bar{\eta}_{4}[\bar{\Psi}]=\bar{\eta}_{4}[\bar{\Phi}]$ in (152).

\subsubsection{Quadratic elements for quasi FLRW solutions encoding STQC structures}

Choosing integration functions resulting in zero N-connection coefficients, we transform (55) into a quasi FLRW metric of type (30),

$$
d s^{2}=\bar{\eta}\left(x^{k}, \tau\right) \stackrel{\circ}{a}^{2}\left(x^{k}, \tau\right)\left\{\bar{\eta}_{i}\left(x^{k}, \tau\right) \stackrel{\circ}{g}_{i}\left[d x^{i}\right]^{2}+\stackrel{\circ}{h}_{3}\left[d y^{3}\right]^{2}\right\}+\stackrel{\circ}{h}_{4}[d \tau]^{2} .
$$

In such a d-metric, the horizontal polarizations $\bar{\eta}_{i}$ are determined by a 2-d Poisson equation with source ${ }_{h} \Upsilon$ as it was explained in (44). The modifications of the cosmological factor $\check{a}^{2}\left(x^{k}, \tau\right)=$ $\bar{\eta}\left(x^{k}, \tau\right) \stackrel{\circ}{a}^{2}\left(x^{k}, \tau\right)$ are given by the polarization $\bar{\eta}\left(x^{k}, \tau\right)$ in (53) determined by solutions of a nonlinear system with source $\Upsilon\left(x^{k}, \tau\right)$ and STQC generating functions $\bar{\Psi}\left(x^{k}, t\right)$ and /or $\bar{\Phi}\left(x^{k}, t\right)$.

We can consider subclasses of generating functions and sources depending only on time like coordinate, when and construct subclasses of solutions of type

$$
d s^{2}=\bar{\eta}(\tau) \stackrel{\circ}{a}^{2}(\tau)\left\{\bar{\eta}_{i}(\tau) \stackrel{\circ}{g}_{i}\left[d x^{i}\right]^{2}+\stackrel{\circ}{h}_{3}\left(d y^{3}\right)^{2}\right\}+\stackrel{\circ}{h}_{4}(d \tau)^{2} .
$$

Such diagonal metrics describe cosmological spacetimes with TC strucure or with memory of STQC, TQC and other type nonlinear interactions. We can chose nonlinear coordinates when $\bar{\eta}_{i}(\tau) \simeq$ $1+\varepsilon \check{\chi}_{i}(\tau)$ as in (36).

Using effective scale factors $\check{a}^{2}\left(x^{k}, \tau\right)$ and/or $\check{a}^{2}(\tau)$, respectively, in (56) and/or (57), we can introduce the respective effective and locally anisotropically polarized Hubble parameters,

$$
\begin{aligned}
\check{H}:=\partial(\ln \check{a}) / \partial \tau= & (\ln \check{a})^{\bullet}=(\ln \stackrel{\circ}{a})^{\bullet}+(\ln \sqrt{|\bar{\eta}|})^{\bullet}, \\
= & \stackrel{\circ}{H}+{ }^{\eta} H, \text { for } \stackrel{\circ}{H}:=(\ln \stackrel{\circ}{a})^{\bullet} \text { and }{ }^{\eta} H:=(\ln \sqrt{|\bar{\eta}|})^{\bullet} .
\end{aligned}
$$

Such a $\check{H}\left(x^{k}, \tau\right)$, or $\check{H}(\tau)$, encodes information on gravitational and matter field STQC structures determined by corresponding classes of dynamical and evolution equations. Cosmological (off-) diagonal evolution in MGTs and GR is defined by polarization $\bar{\eta}=\bar{\eta}_{3}$ computed by formula

$$
\begin{aligned}
\bar{\eta}\left(x^{k}, \tau\right) & =h_{3}^{[0]}\left(x^{k}\right)-\frac{\Phi^{2}\left(x^{k}, \tau\right)}{4 \Lambda} \text { or } \\
\bar{\eta}(\tau) & =h_{3}^{[0]}-\frac{\Phi^{2}(\tau)}{4 \Lambda}, h_{3}^{[0]}=\text { const, for deformations of flat FLRW metrics, } \stackrel{\circ}{h}_{4}=-1
\end{aligned}
$$

Other polarizations describe, in general, a locally anisotropic and/or inhomogeneous gravitational background. In these formulas, the generating $\Upsilon$-sources are prescribed for (effective) matter and 
STQC fields and the polarization/function $\bar{\eta}_{4}$ determine nonlinear gravitational interactions as solutions of (modified) Einstein equations.

We can compute nonholonomic deformations of the prime Hubble radius $\stackrel{\circ}{R}_{H}$ to a target effective Hubble radius $\check{R}_{H}$,

$$
\stackrel{\circ}{R}_{H}:=\frac{1}{\stackrel{\circ}{a} \stackrel{H}{R}} \rightarrow \check{R}_{H}:=\frac{1}{\check{a} \check{H}}=\stackrel{\circ}{R}_{H} \frac{1}{\sqrt{|\bar{\eta}|}+(\sqrt{|\bar{\eta}|})^{\bullet} / \stackrel{\circ}{H}} .
$$

The polarization $\bar{\eta}$ modifies the conditions $\left(\check{R}_{H} \bullet<0\right.$ implying $\left.\check{a}^{\bullet \bullet}>0\right)$ that have to be satisfied in order for inflation (also called early-time accelerating era) to occur in a STQC media.

\subsection{Examples of STQC, time QC and TC solutions}

In this subsection, we compute in explicit form certain examples of conformal factor polarizations generating STQC structures.

\subsubsection{Gravitational STQC structures generated by (effective) matter fields}

Any matter type source $\Upsilon$ modifies nonholonomically a prime cosmological factor $\stackrel{\circ}{a}$ and could generate quasiperiodic space-time structures for certain well-defined conditions. Let us analyze some classes of such solutions:

1. STQC from standard matter in GR:

A source ${ }^{m} \boldsymbol{\Upsilon}_{\mu \nu}=\left(2 M_{P}^{2}\right)^{-1}{ }^{m} \mathbf{T}_{\mu \nu}$ (21) [we can consider, for instance, ${ }^{m} \mathbf{T}_{\mu \nu}$ for a scalar field] defines a STQC polarization of type (59),

$$
\bar{\eta}\left(x^{k}, \tau\right)=\bar{\eta}=\stackrel{\circ}{h}_{3}^{-1}\left(h_{3}^{[0]}-\int d t \frac{\left(\bar{\Psi}^{2}\right)}{4^{m} \Upsilon}\right)=\stackrel{\circ}{h}_{3}^{-1}\left(h_{3}^{[0]}-\frac{\bar{\Phi}^{2}}{4^{m} \Lambda}\right),
$$

if the generating functions (49) are solutions of corresponding STQC equations. We can model such configurations for different choices of functionals for generating functions and $\stackrel{\circ}{h}_{4}=-1$,

$$
\bar{\eta}_{3}=h_{3}^{[0]}\left(x^{k}\right)-\int d \tau \frac{\left(\check{\Psi}^{2}\right)}{4^{m} \check{\Upsilon}}, \text { where }\left\{\begin{array}{ccc}
\check{\Psi}=\check{\Psi}[\bar{\zeta}], & 1 \mathrm{TC}, & \left.\bar{\varsigma}\left(x^{i}, \tau\right) \text { from ([6) }\right) \\
\check{\Psi}=\check{\Psi}[\bar{\chi}, \bar{q}], & 2 \mathrm{TC}, & \bar{\chi}\left(x^{i}, \tau\right), \bar{q}\left(x^{i}, \tau\right) \text { from (19); } \\
\check{\Psi}=\check{\Psi}[\bar{\psi}], & \text { QC, } & \bar{\psi}\left(x^{i}, \tau\right) \text { from (111) } \\
\check{\Psi}=\check{\Psi}[\bar{\chi}, \bar{q}], & \text { mix QC,TCQ, }, & \bar{\chi}\left(x^{i}, \tau\right), \bar{q}\left(x^{i}, \tau\right) \text { from(12) }
\end{array}\right.
$$

We model a cosmological evolution only with time dependent d-metrics with memory on STQC structure of nonlinear interactions if we consider generation functions, or limits to configurations, for

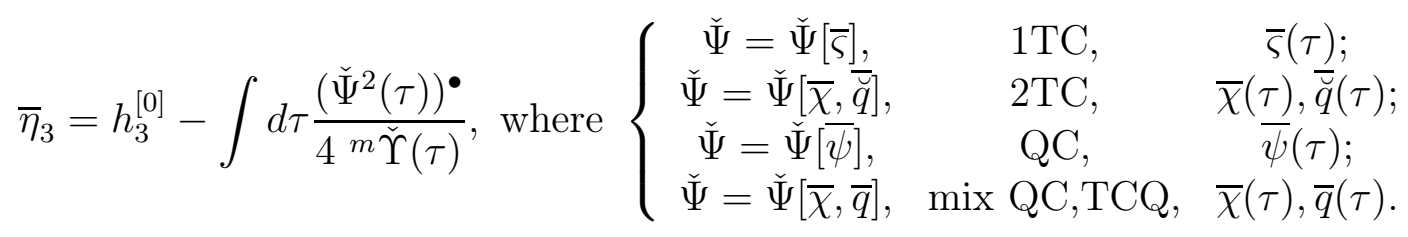

The integration constants can be prescribed in certain forms when there are generated observational quasiperiodic and pattern-forming structures. 


\section{STQC from effective sources in MGTs:}

A $F(R)$ modification of GR induces an effective matter source ${ }^{F} \Upsilon_{\mu \nu}$ (20) which results in STQC polarizations of type (59),

$$
\bar{\eta}\left(x^{k}, \tau\right)=\bar{\eta}=\stackrel{\circ}{h}_{3}^{-1}\left(h_{3}^{[0]}-\int d t \frac{\left(\bar{\Psi}^{2}\right)}{4^{F} \Upsilon}\right)=\stackrel{\circ}{h}_{3}^{-1}\left(h_{3}^{[0]}-\frac{\bar{\Phi}^{2}}{4^{F} \Lambda}\right),
$$

if the generating functions in (44) are solutions of corresponding STQC equations. In explicit form, such modifying gravity generating functions can be computed for $\stackrel{\circ}{h}_{4}=-1$,

$$
\bar{\eta}_{3}=h_{3}^{[0]}\left(x^{k}\right)-\int d \tau \frac{\left(\bar{\Psi}^{2}\right)^{\bullet}}{4{ }^{F} \Upsilon}, \text { where }\left\{\begin{array}{ccc}
\bar{\Psi}=\Psi[\bar{\zeta}], & 1 \mathrm{TC}, & \bar{\varsigma}\left(x^{i}, \tau\right) ; \\
\bar{\Psi}=\Psi[\bar{\chi}, \overline{\breve{q}}], & 2 \mathrm{TC}, & \bar{\chi}\left(x^{i}, \tau\right), \overline{\breve{q}}\left(x^{i}, \tau\right) ; \\
\bar{\Psi}=\Psi[\bar{\psi}], & \text { QC, } & \bar{\psi}\left(x^{i}, \tau\right) ; \\
\bar{\Psi}=\Psi[\bar{\chi}, \bar{q}], & \text { mix QC,TCQ, } & \bar{\chi}\left(x^{i}, \tau\right), \bar{q}\left(x^{i}, \tau\right)
\end{array}\right.
$$

Such configurations are, in general, with nontrivial canonical torsion $\widehat{\mathcal{T}}=0$. Chosing generating functions $\breve{\Psi}$ and considering limits $\widehat{\mathbf{D}}_{\mid \widehat{\mathcal{T}}=0}=\nabla$, we can select LC-configurations for cosmological solutions in MGT. Time $\tau$-depending metrics can be generated for configurations with $\bar{\varsigma}(\tau) ; \bar{\chi}(\tau), \bar{q}(\tau) ; \bar{\psi}(\tau)$; or $\bar{\chi}(\tau), \bar{q}(\tau)$.

\section{Gravitational STQC configurations from quasiperiodic matter :}

A d-metric $\overline{\mathbf{g}}_{\alpha \beta}\left[x^{i}, y^{a} ; \bar{\varsigma}, \bar{\chi}, \bar{q}, \bar{\psi}, \bar{q}, \ldots\right]$ (13) can be generated by a source $\overline{\mathbf{\Upsilon}}_{\mu \nu}=\frac{1}{2 M_{P}^{2}} \overline{\mathbf{T}}_{\mu \nu}$ (21) even generating functions are prescribed not in a STQC form. Such polarizations functions (59) are determined by $\eta_{3}\left(x^{i}, y^{a}\right)$ and/or $\Psi\left(x^{i}, y^{a}\right)$, respectively, $\eta_{3}(\tau)$ and/or $\Psi(\tau)$,

$$
\begin{aligned}
\bar{\eta}_{3}\left(x^{k}, \tau\right) & =h_{3}^{[0]}\left(x^{k}\right)-\int d \tau \frac{\left[\Psi^{2}\left(x^{i}, \tau\right)\right]^{\bullet}}{4 \bar{\Upsilon}\left[x^{i}, \tau ; \bar{\varsigma}, \bar{\chi}, \bar{q}, \bar{\psi}, \bar{q}\right]}, \text { or } \\
\bar{\eta}_{3}(\tau) & =h_{3}^{[0]}-\int d \tau \frac{\left[\Psi^{2}(\tau)\right]^{\bullet}}{4 \bar{\Upsilon}[\tau ; \bar{\varsigma}(\tau), \bar{\chi}(\tau), \bar{q}(\tau), \bar{\psi}(\tau), \bar{q}(\tau)]}
\end{aligned}
$$

for deformations of flat FLRW space-times with $\stackrel{\circ}{h}_{4}=-1$.

LC-conditions on this class of d-metric should be imposed and solved additionally.

\subsubsection{Interacting STQC gravitational and matter fields}

Let us analyze two classes of of nonholonomic deformations:

- In formulas (61), we can introduce STQC generating functions (60) instead of $\Psi\left(x^{i}, \tau\right)$ and compute

$$
\begin{aligned}
\bar{\eta}_{3}\left(x^{k}, \tau\right)= & h_{3}^{[0]}\left(x^{k}\right)-\int d \tau \frac{\left(\bar{\Psi}^{2}\left[\bar{\zeta}\left(x^{i}, \tau\right), \bar{\chi}\left(x^{i}, \tau\right), \bar{q}\left(x^{i}, \tau\right), \bar{\psi}\left(x^{i}, \tau\right), \bar{q}\left(x^{i}, \tau\right)\right]\right)}{4 \bar{\Upsilon}\left[\bar{\varsigma}\left(x^{i}, \tau\right), \bar{\chi}\left(x^{i}, \tau\right), \bar{q}\left(x^{i}, \tau\right), \bar{\psi}\left(x^{i}, \tau\right), \bar{q}\left(x^{i}, \tau\right)\right]}, \text { or } \\
\bar{\eta}_{3}(\tau)= & h_{3}^{[0]}-\int d \tau \frac{\bar{\Psi}^{2}[\bar{\zeta}(\tau), \bar{\chi}(\tau), \bar{q}(\tau), \bar{\psi}(\tau), \bar{q}(\tau)] \bullet}{4 \bar{\Upsilon}[\bar{\varsigma}(\tau), \bar{\chi}(\tau), \bar{q}(\tau), \bar{\psi}(\tau), \bar{q}(\tau)]} \\
& \text { for deformations of flat FLRW space-times with } \stackrel{\circ}{h}_{4}=-1 .
\end{aligned}
$$


- We can study STQC gravitational interactions with effective cosmological constant $\Lambda={ }^{F} \Lambda+$ ${ }^{m} \Lambda+\bar{\Lambda}$ as in (44)

$$
\begin{aligned}
\bar{\eta}_{3}\left(x^{k}, \tau\right) & =\stackrel{\circ}{h}_{3}^{-1}\left(h_{3}^{[0]}-\frac{\bar{\Phi}^{2}\left[\bar{\varsigma}\left(x^{i}, \tau\right), \bar{\chi}\left(x^{i}, \tau\right), \overline{\breve{q}}\left(x^{i}, \tau\right), \bar{\psi}\left(x^{i}, \tau\right), \bar{q}\left(x^{i}, \tau\right)\right]}{4\left({ }^{F} \Lambda+{ }^{m} \Lambda+\bar{\Lambda}\right)}\right) \text { or } \\
\bar{\eta}_{3}(\tau) & =\stackrel{\circ}{h}_{3}^{-1}\left(h_{4}^{[0]}-\frac{\bar{\Phi}^{2}[\bar{\varsigma}(\tau), \bar{\chi}(\tau), \bar{q}(\tau), \bar{\psi}(\tau), \bar{q}(\tau)]}{4\left({ }^{F} \Lambda+{ }^{m} \Lambda+\bar{\Lambda}\right)}\right) .
\end{aligned}
$$

LC-configurations in GR can be extracted from these formulas if we prescribe ${ }^{F} \Lambda=0$ and $\bar{\Phi}[\bar{\varsigma}, \bar{\chi}, \bar{q}, \bar{\psi}, \bar{q}]$ is chosen as in (49) . For such solutions, we can re-define formulas with explicit sources ${ }^{F} \Upsilon+{ }^{m} \Upsilon+\bar{\Upsilon}$ if a corresponding $\bar{\Psi}$-generating function is introduced if there are considered nonlinear symmetries (46).

\subsubsection{A toy model with 1-TQC structure for DM}

A simple example of d-metric $\overline{\mathbf{g}}_{\alpha \beta}\left[x^{i}, y^{a} ; \varsigma, \ldots\right]$ of type (13) can be generated by a source $\overline{\mathbf{\Upsilon}}_{\mu \nu}[\varsigma]=$ $\frac{1}{2 M_{P}^{2}} \overline{\mathbf{T}}_{\mu \nu}[\varsigma]$, see formulas (21) and (17), computed for a DM Lagrange density $\overline{\mathcal{L}}=\dot{L}(\varsigma)$, see formulas (16) and (5). In such a toy model, the generating functions are prescribed in a general but not in a STQC form. Nevertheless, the polarizations functions (59) are determined by $\eta_{3}\left(x^{i}, y^{a}\right)$ and/or $\Psi\left(x^{i}, y^{a}\right)$, respectively, $\eta_{3}(\tau)$ and/or $\Psi(\tau)$, as a subclass of configurations of type (61) when

$$
\begin{aligned}
\bar{\eta}_{3}\left[x^{k}, \tau ; \varsigma\left(x^{k}, \tau\right)\right] & =h_{3}^{[0]}\left(x^{k}\right)-\int d \tau \frac{\left[\Psi^{2}\left(x^{i}, \tau\right)\right]}{4 \bar{\Upsilon}\left[x^{i}, \tau ; \varsigma\right]}, \text { or } \\
\bar{\eta}_{3}[\tau ; \varsigma(\tau)] & =h_{3}^{[0]}-\int d \tau \frac{\left[\Psi^{2}(\tau)\right]^{\bullet}}{4 \bar{\Upsilon}[\tau ; \varsigma(\tau)]}
\end{aligned}
$$

for deformations of flat FLRW space-times with $\stackrel{\circ}{h}_{4}=-1$.

We can impose the LC-conditions on this class of d-metric and generate such 1-TQC configurations for DM in the framework of GR.

Off-diagonal and diagonalized cosmological metrics with gravitational polarizations of type (63) results in nontrivial inflation scenarios as we conclude below in subsection 4.2.2. Such values of $\bar{\eta}_{3}[\varsigma]$ can be used also for elaborating examples of toy models of a late 1-TQC dynamics with some general DE but just 1-TQC configurations for DM. We should reproduced for such a particular choice all constructions from section [5] where the approach is elaborated for a general STQC dynamics. The simplified scenarios of 1-TQC acceleration and DM are useful in understanding possible physical implications of cosmological solutions with time quaisperiodic structures both in MGTs and GR. Nevertheless, modern cosmological data [37, 38] state a more complex nonlinear structure and evolution of Universe which can be described more realistically if there are used 2-TQC and more sophisticate time and space like quasiperiodic configurations and interactions of gravitational and (effective) matter fields.

\section{Inflationary STQC dynamics}

We studied models of in MGTs and GR for locally anisotropic space-time quasi-periodic and pattern forming structures in [1, 2, 3, 4, 5, 6], see references therein. The $F(R)$ gravity is the most 
popular among MGTs and admits various massive, mimetic, topological gravity and string type generalizations - there are a series of reviews in literature [7, 8, 9, 10, 11, 12, 13, 14, 15, 16, 17]. The goal of this section is to elaborate on inflation cosmology in nonholonomic space-times with STQC structure encoded into an effective cosmological scale factor $\bar{a}^{2}=\bar{\eta} \stackrel{\circ}{ }^{2}$ and corresponding nonholonomic deformations of Hubble constants, $\check{H}=\stackrel{\circ}{H}+{ }^{\eta} H$ (58), with gravitational polarization $\bar{\eta}$ (59). We can always chose generating functions or find limits to configurations with $\check{H}(\tau)=\stackrel{\circ}{H}(\tau)+{ }^{\eta} H(\tau)$ and $\bar{\eta}(\tau)$ encoding prescribed STQC structures as we described in previous section. These values can be used for modeling inflation scenarios in off-diagonal backgrounds with nonholonomic N-connection structures and/or "memory" of nonlinear gravitational and matter field interactions contained into diagonalized quasi FLRW metrics (30).

\subsection{General cosmological properties of gravity theories with STQC struc- ture}

\subsubsection{Perfect fluid representation of STQC configurations in GR}

We start with the quasi FLRW configurations in GR coupled with an effective perfect fluid modelling STQC structures (with nontrivial effective sources ${ }^{m} \Upsilon$ and $\bar{\Upsilon}$ but ${ }^{F} \Upsilon=0$, from for gravitational field equations (18) $)$. With respect to N-adapted frames, we have (see (19) $)$

$$
T_{\alpha \beta} \sim \operatorname{diag}\left[\check{\eta}_{i}(\tau){ }^{e} p,{ }^{e} p,-{ }^{e} p\right]
$$

for respective components of effective pressure, ${ }^{e} p={ }^{m} p+\bar{p}$, and effective energy density, ${ }^{e} \rho=$ ${ }^{m} \rho+\bar{\rho}$, components. The polarizations $\bar{\eta}_{i}(\tau) \simeq 1+\varepsilon \bar{\chi}_{i}(\tau)$ can be chosen and treated as small anisotropies for diagonalized d-metrics, see (36). For such parameterizations, the effective FLRW equations for diagonalized target d-metrics are

$$
\begin{aligned}
& { }^{m} p+\bar{p}=\frac{3}{2 M_{P}^{2}} \check{H}^{2}=\frac{3}{2 M_{P}^{2}}\left(\stackrel{\circ}{H}+{ }^{\eta} H\right)^{2} \\
& { }^{m} \rho+\bar{\rho}=-\frac{1}{2 M_{P}^{2}}\left[3 \check{H}^{2}+2 \check{H}^{\bullet}\right]=-\frac{1}{2 M_{P}^{2}}\left[3\left(\stackrel{\circ}{H}+{ }^{\eta} H\right)^{2}+2\left(\stackrel{\circ}{H}+{ }^{\eta} H\right)^{\bullet}\right] .
\end{aligned}
$$

In GR, it is also used the gravitational constant $\kappa^{2}=2 M_{P}^{2}$. The effective equation of state (EoS) is computed

$$
{ }^{e} w=-1-2 \check{H}^{\bullet} / 3 \check{H}^{2}=-1-2\left(\stackrel{\circ}{H}+{ }^{\eta} H\right)^{\bullet} / 3\left(\stackrel{\circ}{H}+{ }^{\eta} H\right)^{2} .
$$

Such formulas encode STQC configurations even if quasi-periodic sources of matter are with $\bar{\rho}=0$ and $\bar{p}=0$ but nontrivial values ${ }^{\eta} H$ are defined by gravitational STQC interactions

\subsubsection{Analogous perfect fluid representation of STQC structures in MGT}

For flat quasi FLRW metrics (55), the modified gravity equations (18) with sources (19) results in FLRW equations (see details in 11, 2, 3, 4, 6); for simplicity, we use only the effective Hubble rate $\check{H}$ and $\mathbf{F}(\widehat{\mathbf{R}})$ and it functional derivatives on $\widehat{\mathbf{R}} \simeq 12 \check{H}^{2}+6 \check{H}^{\bullet}, \check{H}^{\bullet}=\partial \check{H} / \partial \tau$, which can subjected 
to additional LC-conditions),

$$
\begin{aligned}
2 M_{P}^{2}\left({ }^{m} p+\bar{p}\right)= & -36\left[\check{H}^{\bullet \bullet}+4 \check{H} \check{H}^{\bullet}\right]^{2} \mathbf{F}^{\prime \prime \prime}(\widehat{\mathbf{R}})-6\left[\check{H}^{\bullet \bullet \bullet}+6 \check{H}^{\bullet \bullet} \check{H}+4(\check{H} \bullet)^{2}+8 \check{H}^{2} \check{H}^{\bullet}\right] \mathbf{F}^{\prime \prime}(\widehat{\mathbf{R}}) \\
& +\left(3 \check{H}^{2}+\check{H}^{\bullet}\right) \mathbf{F}^{\prime}(\widehat{\mathbf{R}})-\frac{1}{2} \mathbf{F}(\widehat{\mathbf{R}}), \\
2 M_{P}^{2}\left({ }^{m} \rho+\bar{\rho}\right)= & 18\left[\check{H}^{\bullet \bullet} \check{H}+4\left(\check{H}^{\bullet}\right)^{2}\right] \mathbf{F}^{\prime \prime}(\widehat{\mathbf{R}})-3\left(\check{H}^{\bullet}+\check{H}^{2}\right) \mathbf{F}^{\prime}(\widehat{\mathbf{R}})+\frac{1}{2} \mathbf{F}(\widehat{\mathbf{R}}) .
\end{aligned}
$$

These equations can be represented in effective form (64),

$$
\check{p}=\frac{3}{2 M_{P}^{2}} \check{H}^{2} \text { and } \check{\rho}=-\frac{1}{2 M_{P}^{2}}\left(3 \check{H}^{2}+2 \check{H}^{\bullet}\right),
$$

when the respective values are computed for $\mathbf{F}(\widehat{\mathbf{R}})=\widehat{\mathbf{R}}+\check{\mathbf{F}}(\widehat{\mathbf{R}})$,

$$
\begin{aligned}
2 M_{P}^{2} \check{p}= & 2 M_{P}^{2}\left({ }^{m} p+\bar{p}\right)+36\left[\check{H}^{\bullet \bullet}+4 \check{H} \check{H}^{\bullet}\right]^{2} \check{\mathbf{F}}^{\prime \prime \prime}(\widehat{\mathbf{R}})+6\left[\check{H}^{\bullet \bullet \bullet}+6 \check{H}^{\bullet \bullet} \check{H}+4\left(\check{H}^{\bullet}\right)^{2}\right. \\
& \left.+8 \check{H}^{2} \check{H}^{\bullet}\right] \check{\mathbf{F}}^{\prime \prime}(\widehat{\mathbf{R}})-\left(3 \check{H}^{2}+\check{H}^{\bullet}\right) \check{\mathbf{F}}^{\prime}(\widehat{\mathbf{R}})+\frac{1}{2} \check{\mathbf{F}}(\widehat{\mathbf{R}}), \\
2 M_{P}^{2} \check{\rho}= & 2 M_{P}^{2}\left({ }^{m} \rho+\bar{\rho}\right)-18\left[\check{H}^{\bullet \bullet} \check{H}+4\left(\check{H}^{\bullet}\right)^{2}\right] \check{\mathbf{F}}^{\prime \prime}(\widehat{\mathbf{R}})+3\left(\check{H}^{\bullet}+\check{H}^{2}\right) \check{\mathbf{F}}^{\prime}(\widehat{\mathbf{R}})-\frac{1}{2} \check{\mathbf{F}}(\widehat{\mathbf{R}}) .
\end{aligned}
$$

For LC-configurations, these formulas admit a scalar tensor description and can be used elaborating viable dark energy models, see review of works in [8]. In [1, 3, 6], there are studied off-diagonal generalizations for locally anisotropic cosmological models with possible massive gravity and QC generalizations.

\subsubsection{Non-minimal coupling of MGT and STQC configurations}

We studied locally anisotropic cosmological configurations and/or QC structures in MGTs with non-minimal coupling in [2, 6]. In this work, we elaborate on modified cosmology model with STQC structure defined by action

$$
{ }^{f} \mathcal{S}=\int d^{4} u \sqrt{|\mathbf{g}|}\left\{\frac{M_{P}^{2}}{2} \mathbf{F}(\widehat{\mathbf{R}})+\mathbf{f}(\widehat{\mathbf{R}})\left({ }^{m} \mathcal{L}+\overline{\mathcal{L}}\right)\right\}
$$

where $\mathbf{f}(\widehat{\mathbf{R}})$ is a functional on $\widehat{\mathbf{R}}$ and Lagrange densities are used for definition of energy-momentum tensors for usual matter and STQC matter sources (21), ${ }^{m} \mathbf{T}_{\mu \nu}$ and $\overline{\mathbf{T}}_{\mu \nu}$. Applying a N-adapted variational calculus for ${ }^{f} \mathcal{S}$, we obtain the gravitational field equations

$$
\left.\widehat{\mathbf{R}}_{\mu \nu} \mathbf{F}^{\prime}(\widehat{\mathbf{R}})+\left[\mathbf{g}_{\mu \nu} \widehat{\mathbf{D}}^{\tau} \widehat{\mathbf{D}}_{\tau}-\frac{1}{2}\left(\widehat{\mathbf{D}}_{\mu} \widehat{\mathbf{D}}_{\nu}+\widehat{\mathbf{D}}_{\nu} \widehat{\mathbf{D}}_{\mu}\right)\right]\right) \mathbf{F}^{\prime}(\widehat{\mathbf{R}})-\frac{1}{2} \mathbf{g}_{\mu \nu} \mathbf{F}(\widehat{\mathbf{R}})=\frac{1}{2 M_{P}^{2}}{ }^{f} \mathbf{T}_{\mu \nu}
$$

where the effective energy-momentum tensor is defined

$$
{ }^{f} \mathbf{T}_{\mu \nu}:=\left[{ }^{m} \mathbf{T}_{\mu \nu}+\overline{\mathbf{T}}_{\mu \nu}-\widehat{\mathbf{R}}_{\mu \nu}\left({ }^{m} \mathcal{L}+\overline{\mathcal{L}}\right)\right] \mathbf{f}^{\prime}(\widehat{\mathbf{R}})+\left[\mathbf{g}_{\mu \nu} \widehat{\mathbf{D}}^{\tau} \widehat{\mathbf{D}}_{\tau}-\frac{1}{2}\left(\widehat{\mathbf{D}}_{\mu} \widehat{\mathbf{D}}_{\nu}+\widehat{\mathbf{D}}_{\nu} \widehat{\mathbf{D}}_{\mu}\right)\right] \mathbf{f}^{\prime}(\widehat{\mathbf{R}})
$$


In the approximation of effective perfect fluid modelling matter field interactions and STQC structures, we define the $f$-effective pressure and energy density,

$$
\begin{aligned}
{ }^{f} p:= & \frac{\partial^{2}}{\partial \tau^{2}}\left[\mathbf{f}^{\prime}(\widehat{\mathbf{R}})\left({ }^{m} \mathcal{L}+\overline{\mathcal{L}}\right)\right]+4 \check{H} \frac{\partial}{\partial \tau}\left[\mathbf{f}^{\prime}(\widehat{\mathbf{R}})\left({ }^{m} \mathcal{L}+\overline{\mathcal{L}}\right)\right] \\
& -\left(\check{H}^{\bullet}+3 \check{H}^{2}\right) \mathbf{f}^{\prime}(\widehat{\mathbf{R}})\left({ }^{m} \mathcal{L}+\overline{\mathcal{L}}\right)+\mathbf{f}(\widehat{\mathbf{R}})\left({ }^{m} p+\bar{p}\right), \\
{ }^{f} \rho:= & -3 \check{H} \frac{\partial}{\partial \tau}\left[\mathbf{f}^{\prime}(\widehat{\mathbf{R}})\left({ }^{m} \mathcal{L}+\overline{\mathcal{L}}\right)\right]+3\left(\check{H}^{\bullet}+\check{H}^{2}\right) \mathbf{f}^{\prime}(\widehat{\mathbf{R}})\left({ }^{m} \mathcal{L}+\overline{\mathcal{L}}\right)+\mathbf{f}(\widehat{\mathbf{R}})\left({ }^{m} \rho+\bar{\rho}\right),
\end{aligned}
$$

where $\left({ }^{m} p+\bar{p}\right)$ and $\left({ }^{m} \rho+\bar{\rho}\right)$ are defined by ${ }^{m} \mathbf{T}_{\mu \nu}+\overline{\mathbf{T}}_{\mu \nu}$.

It should be noted that models with non-trivial coupling of type ${ }^{f} \mathcal{S}$ can be elaborated for describing accelerating expansion (in general, in locally anisotropic form) of the Universe, see details and reviews of results in [8, 2, 6].

\subsection{Effective scalar field and STQC inflation}

The inflationary paradigm solved the problems of Big Bang cosmology by adding a period of nearly exponential expansion during early time cosmological evolution. In the literature there many historical and comprehensive reviews for various MGTs and cosmology see [7, 8, 19, 10, 11, 12, 13, 14, [15, 16, 17] and references therein. In the simplest approach, such inflation scenarios were modeled by homogeneous solutions of (modified) Einstein and nonlinear Klein-Gordon equations for scalar fields.

In this subsection, we provide a condensed description and analyze some concrete toy models of inflation with nontrivial STQC structure. For simplicity, we assume that the inflationary dynamics is controlled by certain nonlinear gravitational and matter field interactions resulting in STQC configurations when the main issues can be addressed in some diagonal parameterizations or approximations for a perfect (effective) matter fluid encoding time and space quasiperiod structures and memory of off-diagonal nonlinear interactions. For small parametric constructions with quasi FLRW metrics (33)-(36), our approach can be related to cosmological perturbation theories but we do not discuss such details here.

\subsubsection{Scalar field description and gravitational STQC inflation}

Consider a scalar field with $\phi\left(x^{i}, \tau\right)$ with action (14) for ${ }^{m} \mathcal{L}=\frac{1}{2}\left(\mathbf{e}_{\alpha} \phi\right)\left(\mathbf{e}^{\alpha} \phi\right)+V(\phi)$ and compute parameterize the energy-momentum tensor ${ }^{m} \mathbf{T}_{\alpha \beta}$ (15) for a diagonalized metric (57) with $\bar{\eta}_{i}(\tau) \simeq$ $1+\varepsilon \check{\chi}_{i}(\tau)$, as follow

$$
{ }^{m} \mathbf{T}_{j}^{i}=p \delta_{\grave{j}}^{i}, \text { for } p=\frac{1}{2}\left(\phi^{\bullet}\right)^{2}-V(\phi),{ }^{m} \mathbf{T}_{4}^{4}=-\rho=\frac{1}{2}\left(\phi^{\bullet}\right)^{2}+V(\phi) .
$$

These formulas result in modified Friedmann equations $\frac{3}{2 M_{P}^{2}} \check{H}^{2}=\rho$. Taking the first derivative on $\tau$, considering previous formula for $\rho$, and $\check{H}^{\bullet}=-M_{P}^{2}\left(\phi^{\bullet}\right)^{2}$, we obtain a nonlinear modification of the Klein-Gordon equation for the canonical scalar filed $\phi$ in a nonholonomic cosmological background,

$$
\phi^{\bullet \bullet}+3 \check{H} \phi^{\bullet}+\partial V / \partial \phi=0 .
$$


This allows us to compute gravitational STQC modifications of inflation of the slow-roll indices ${ }^{\text {inf } \epsilon}$ and ${ }^{\inf } \eta$ (we use left abstract labels "inf" from inflation in order to avoid ambiguities with other type values also denoted by $\epsilon$ and, respectively, $\eta$ ),

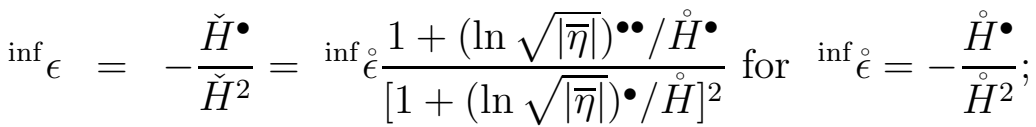

$$
\begin{aligned}
& { }^{\inf } \eta=-\frac{\check{H}^{\bullet \bullet}}{2 \check{H} \check{H}^{\bullet}}=\inf _{\eta} \frac{1+(\ln \sqrt{|\bar{\eta}|})^{\bullet \bullet \bullet} / \stackrel{\circ}{H}^{\bullet \bullet}}{\left.\left.2\left[1+(\ln \sqrt{|\bar{\eta}|})^{\bullet} / \stackrel{\circ}{H}\right)\right][1+(\ln \sqrt{|\bar{\eta}|}))^{\bullet \bullet} / \stackrel{\circ}{H}^{\bullet}\right]} \text {, for } \quad \inf \stackrel{\circ}{\eta}=-\frac{\stackrel{\circ}{H}^{\bullet \bullet}}{2 \stackrel{\circ}{H} \stackrel{\circ}{H}^{\bullet}} \text {. }
\end{aligned}
$$

The slow-roll conditions for inflation state that ${ }^{\inf } \epsilon, \inf _{\epsilon}^{\circ},{ }^{\inf } \eta,{ }^{\inf } \stackrel{\circ}{\eta} \ll 1$, when $\phi^{\bullet \bullet} \ll \check{H} \phi^{\bullet}$ and $\phi^{\bullet \bullet} \ll \stackrel{\circ}{H} \phi^{\bullet}$, the modified Friedman and Klein equations, and respective formulas for slow-roll indices, are simplified as follows,

$$
\begin{aligned}
\check{H}^{2} & \simeq \frac{2 M_{P}^{2}}{3} V \text { and } 3 \check{H} \phi \bullet-\partial V / \partial \phi \\
\inf _{\epsilon} & =-M_{P}^{2} \frac{\left(\phi^{\bullet}\right)^{2}}{\check{H}^{2}},{ }^{\inf } \stackrel{\circ}{\eta}=2 M_{P}^{2} \frac{\left(\phi^{\bullet}\right)^{2}}{\check{H}^{2}}+2 \frac{\phi^{\bullet \bullet}}{\check{H} \phi} .
\end{aligned}
$$

Using pervious formulas, we can compute the relations between prime and target slow-roll indices and the potential $V(\phi)$ following formulas,

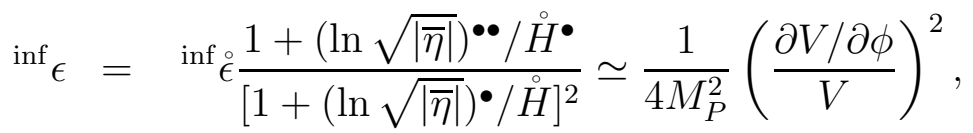

$$
\begin{aligned}
& { }^{\inf } \eta=\inf _{\eta} \frac{1+(\ln \sqrt{|\bar{\eta}|})^{\bullet \bullet \bullet} / \stackrel{\circ}{H}^{\bullet \bullet}}{2[1+(\ln \sqrt{|\bar{\eta}|}) \bullet / \stackrel{\circ}{H})]\left[1+(\ln \sqrt{|\bar{\eta}|})^{\bullet \bullet} / \stackrel{\circ}{H}^{\bullet}\right]} \simeq \frac{1}{2 M_{P}^{2}} \frac{\left|\partial^{2} V / \partial^{2} \phi\right|}{V}
\end{aligned}
$$

The modified graceful exit from the inflationary era occurs when $\inf _{\epsilon} \sim O(1)$. Using formulas (66), we can compute nonholonomic deformations by gravitational STQC structures of the spectral indices from a prime state into target one, for

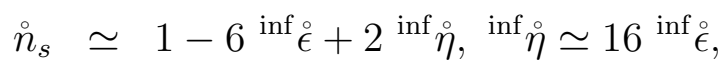

$$
\begin{aligned}
& n_{s} \simeq 1-6{ }^{\mathrm{inf}} \epsilon+2{ }^{\mathrm{inf}} \eta,{ }^{\mathrm{inf}} \eta \simeq 16{ }^{\mathrm{inf}} \epsilon \text {, }
\end{aligned}
$$

calculated at the horizon crossing. We can consider various cosmological configurations with nontrivial vacuum structure determined by a gravitational polarization $\bar{\eta}\left[x^{i}, y^{a} ; \bar{\varsigma}, \bar{\chi}, \bar{q}, \bar{\psi}, \bar{q}, \ldots\right]$. For corresponding functional dependencies, such configurations may induce an inflationary era, or to result in a graceful exit.

\subsubsection{Inflation determined by a scalar field with TC structure}

We can consider instead the Lagrangian for a scalar filed the Lagrange density (6) . In the nonrelativistic approximation $\varsigma \rightarrow \varsigma(t)$, we can summarize the motion equations and the Klein-Gordon equations for $\varsigma(t)$ and consider a nonlinear equation,

$$
\left(\varsigma^{\bullet}\right)^{2} \varsigma^{\bullet}+3 \check{H} \varsigma^{\bullet}+2 \frac{\partial \dot{V}^{\prime}}{\partial \varsigma}=0
$$


A subclass of solutions can be constructed for $\varsigma^{\bullet} \simeq 1+\varepsilon \chi^{\bullet}$ with a small parameter $\varepsilon$ and $V^{\prime} \sim \varepsilon V(\chi)$, which results in

$$
\chi^{\bullet \bullet}+3 \check{H} \chi \bullet+\partial Z(\chi) / \partial \chi=0,
$$

with redefined potential $\partial Z(\chi) / \partial \chi=2 \frac{\partial V(\chi)}{\partial \chi}+3 \check{H}$. In result, we obtain modifications of the inflation slow-roll parameters (66) by an additional TC structure,

$$
\begin{aligned}
& \inf _{\epsilon}=\inf _{\epsilon} \frac{1+(\ln \sqrt{|\bar{\eta}|})^{\bullet \bullet / \stackrel{\circ}{\bullet}}}{\left[1+(\ln \sqrt{|\bar{\eta}|})^{\bullet} / \stackrel{\circ}{H}\right]^{2}} \simeq \frac{1}{4 M_{P}^{2}}\left(\frac{\partial Z / \partial \chi}{Z}\right)^{2} \\
& { }^{\inf } \eta=\inf _{i} \frac{1+(\ln \sqrt{|\bar{\eta}|})^{\bullet \bullet \bullet} / \stackrel{\circ}{H}^{\bullet \bullet}}{2[1+(\ln \sqrt{|\bar{\eta}|}) \bullet / \stackrel{\circ}{H})]\left[1+(\ln \sqrt{|\bar{\eta}|})^{\bullet \bullet / \stackrel{\circ}{\bullet}]}\right.} \simeq \frac{1}{2 M_{P}^{2}} \frac{\left|\partial^{2} Z / \partial^{2} \chi\right|}{Z} .
\end{aligned}
$$

For such a model, the inflation era is determined both by an effective one-dimensional time crystal structure for effective scalar fields and nonlinear gravitational fields.

\subsubsection{STQC inflation from $F(R)$ theories}

We analyse slow-roll inflation for an example of MGT with STQC structure when the evolution dynamics is determined by four generalized slow-roll indices. Such values can be introduced and computed for off-diagonal solutions as in [8, 2, 6] using ${ }^{i} \check{H}$ (58),

$$
\begin{aligned}
& \check{\epsilon}_{1}=-\frac{\check{H}^{\bullet}}{\check{H}^{2}}=\stackrel{\circ}{\epsilon}_{1} \frac{1+(\ln \sqrt{|\bar{\eta}|})^{\bullet \bullet} / \stackrel{\circ}{H}^{\bullet}}{\left[1+(\ln \sqrt{|\bar{\eta}|})^{\bullet} / \stackrel{\circ}{H}\right]^{2}}, \text { for } \stackrel{\circ}{\epsilon}_{1}=-\frac{\stackrel{\circ}{H}^{\bullet}}{\check{H}^{2}} \\
& \check{\epsilon}_{2}=0 ; \check{\epsilon}_{3} \simeq-\check{\epsilon}_{1} \text { and } \stackrel{\circ}{\epsilon}_{3} \simeq-\check{\circ}_{1} ; \check{\epsilon}_{4} \simeq-3 \check{\epsilon}_{1}+\check{H}^{-1}\left(\ln \left|\check{\epsilon}_{1}\right|\right)^{\bullet} \text { and } \stackrel{\circ}{\epsilon}_{4} \simeq-3 \stackrel{\circ}{\epsilon}_{1}+\stackrel{\circ}{H}^{-1}\left(\ln \left|\stackrel{\circ}{\epsilon}_{1}\right|\right)^{\bullet} .
\end{aligned}
$$

Such values allow us to compute in the slow-roll limit (when $\check{\epsilon}_{1}, \check{\epsilon}_{4} \ll 1$ ) the nonholonomic $\bar{\eta}^{-}$ deformations to a target gravitational STQC configuration when respective spectral index of primordial curvature perturbations and the scalar-to-tensor ratio can be computed following formulas

$$
\check{n}_{s} \simeq 1-6 \check{\epsilon}_{1}-2 \check{\epsilon}_{4} \text { and } \check{r}=48\left(\check{\epsilon}_{1}\right)^{2} \text {. }
$$

We can generate a viable inflation model for a target configuration even with observational compatible $\check{n}_{s}$ and $\check{r}$ even certain primary data $\stackrel{\circ}{\epsilon}_{1}$ and $\stackrel{\circ}{\epsilon}_{4}$ do not satisfy necessary conditions for inflation era with similar $\stackrel{\circ}{n}_{s}$ and $\stackrel{\circ}{r}$.

The Starobinsky $R^{2}$ model of inflation [36] was studied in a number of cosmological works because it is compatible with Planck data [37, 38] and can explain equivalently a scalar field and a modified gravity model involving a phenomenological constant ${ }^{i} \mathrm{H}$ of dimension of $\left[\mathrm{mass}^{2}\right]$. In hour approach [1, 2, 3, 4, 5, 6], we consider that during an effective slow-roll inflation era

$$
\mathbf{F}(\widehat{\mathbf{R}})=\widehat{\mathbf{R}}+\widehat{\mathbf{R}}^{2} / 36\left({ }^{i} H\right)
$$

where constants a chosen in such a form which allow for LC-configurations to apply the method for solution of FLRW equations following formulas (306)-(316) in [8] and generalized to N-adapted computations in [2, 6]. For a flat quasi FLRW metric (55) with small $h$-polarizations, the Friedman equations are equivalent to

$$
\begin{aligned}
\check{H}^{\bullet \bullet}-\frac{\left(\check{H}^{\bullet}\right)^{2}}{2 \check{H}}+3 \check{H}\left({ }^{i} H+\check{H}^{\bullet}\right) & =0, \\
\widehat{\mathbf{R}}^{\bullet \bullet}+3 \widehat{\mathbf{R}}\left(\check{H}+2{ }^{i} H\right) & =0 .
\end{aligned}
$$


During a slow-roll era, we can neglect the first two terms in the first equation in the system above and find a quasi de Sitter solution for cosmological quasi linear in time $\tau$ evolution when

$$
\check{H}(\tau) \simeq{ }^{0} \check{H}-{ }^{i} H\left(\tau-\tau_{k}\right),
$$

where the STQC nonholonomic deformations are encoded in

$$
{ }^{0} \check{H}={ }^{0} \stackrel{\circ}{H}\left[1+\left(\ln \sqrt{\left|{ }^{0} \bar{\eta}\right|}\right) \bullet /{ }^{0} \stackrel{\circ}{H}\right] .
$$

Here we consider a prime a Hubble constant ${ }^{0} \stackrel{\circ}{H}$ modified by ${ }^{0} \bar{\eta}^{\bullet}\left(\tau_{k}\right)$, when $\tau$ takes values from a time instance $\tau_{k}$, when small-roll approximations hold true, till a moment $\tau=\tau_{f}$ for $\check{\epsilon}_{1}\left(\tau_{f}\right) \simeq 1$, when

$$
\check{H}\left(\tau_{f}\right)=\check{H}_{f} \simeq \sqrt{{ }^{i} H} \text { and } \tau_{f}-\tau_{k} \simeq{ }^{0} \check{H} /{ }^{i} H
$$

Here we note that both ${ }^{0} \breve{H}$ and ${ }^{i} H$ are expected to have large values during inflation era (at least for target models). The effective $e$-folding number is introduced by definition and computed

$$
\check{N}:=\int_{\tau_{k}}^{\tau_{f}} \check{H}(\tau) d \tau \simeq\left({ }^{0} \check{H}\right)^{2} / 2{ }^{i} H .
$$

This number is affected by the STCQ structure via ${ }^{0} \bar{\eta}$, see formula (70). Usually, there are considered large $\check{N}$ limits in the observational formulas.

Using above formulas, we can find STQC deformations of the observational indices (67a) when

$$
\begin{aligned}
\check{n}_{s} & \simeq 1-4{ }^{i} H\left({ }^{0} \check{H}-\frac{2{ }^{i} H \check{N}}{{ }^{0} \check{H}}\right)^{-2} \simeq 1-\frac{2}{\check{N}}, \text { for large } \check{N} \\
& \simeq 1-\frac{2}{{ }^{0} \check{N}\left[1+\left(\ln \sqrt{\left|{ }^{0} \bar{\eta}\right|}\right) \bullet /{ }^{0} \stackrel{\circ}{H}\right]^{2}} \text { for }{ }^{0} \check{N} \simeq\left({ }^{0} \stackrel{\circ}{H}\right)^{2} / 2{ }^{i} H \\
\check{r} & \simeq 48\left({ }^{i} H\right)^{2}\left({ }^{0} \check{H}-\frac{2{ }^{i} H \check{N}}{{ }^{0} \check{H}}\right)^{-4} \simeq \frac{12}{\check{N}^{2}}, \text { for large } \check{N}, \simeq \frac{12}{\left({ }^{0} \check{N}\right)^{2}\left[1+\left(\ln \sqrt{\left|{ }^{0} \bar{\eta}\right|}\right)^{\bullet} /{ }^{0} \stackrel{\circ}{H}\right]^{4}} .
\end{aligned}
$$

In Einstein and Jordan N-adapted frames, we yield the same observation indices at the leading order for target metrics and models. The graceful exit from inflation in such MGTs occurs due to the $R^{2}$ term, it distortion, and STQC polarizations. All such effects may contribute to un-stability of the de Sitter point. The vacuum de Sitter configurations are with memory and STQC structure when nonlinear perturbations could result in unstable locally anisotropic quasi de Sitter attractors. Hence, the graceful exit comes as a result of growing curvature perturbations, nonholonomic deformations (which can make stabile the dynamics for certain special classes of non-integrable constraints) and because of gravitational and matter filed quasi-periodic interactions and pattern-forming structures.

\subsubsection{Reheating and STQC structures in nonholonomic MGTs}

In this section, we discuss in brief how STQC structures affect the reheating period in $R^{2}$ gravity (68) (the most representative modified gravity model) that fills the gap between inflation and the radiation and matter domination era. In our approach, ${ }^{i} H$ is the same as $H_{i}$ in formulas (482)-(498) from review [8]. Those results can be used to study observational indices of inflation, see formulas (172) and (73); the reheating Friedman temperatures. During the reheating era, the term $\widehat{\mathbf{R}}^{\bullet \bullet}$ in (69) 
became important and the evolution of the scalar curvature is driven by an equation which is similar for that for damped harmonic oscillator with restoring force of order $3^{i} H$.

Reheating is a conventional oscillatory era when the terms $\check{H}^{\bullet \bullet}$ and $\frac{(\check{H} \bullet)^{2}}{2 \check{H}}$ dominate but $3 \check{H} \check{H}^{\bullet}$ sub-dominates in the first equation (69). Here we note that during the reheating phase $\widehat{\mathbf{R}} \simeq 6 \check{H}^{\bullet}$ which is in contrast with $\widehat{\mathbf{R}} \simeq 6 \check{H}^{2}$ for the slow-roll era. This results in such solutions for the reheating phases:

$$
\begin{aligned}
\check{a} & =\check{a}_{r}\left[1+\frac{\omega}{4}\left(\tau-\check{\tau}_{r}\right)\right]^{2 / 3}, \check{H} \simeq \frac{8 \omega}{3} \frac{\cos ^{2} \omega\left(\tau-\check{\tau}_{r}\right)}{8+2 \omega\left(\tau-\check{\tau}_{r}\right)+\sin 2 \omega\left(\tau-\check{\tau}_{r}\right)} \\
\widehat{\mathbf{R}} & \simeq \frac{16 \omega}{3} \frac{\sin 2 \omega\left(\tau-\check{\tau}_{r}\right)}{8+2 \omega\left(\tau-\check{\tau}_{r}\right)+\sin 2 \omega\left(\tau-\check{\tau}_{r}\right)},
\end{aligned}
$$

where $\check{a}_{r}=a_{0} \exp \left[\left({ }^{0} \check{H}\right)^{2} / 2{ }^{i} H-1 / 12\right]$ for a scale factor $a_{0}$ corresponding to the onset of inflation, $\omega=\sqrt{3^{i} H / 2}$ and the reheating time $\check{\tau}_{r} \simeq\left({ }^{0} \check{H}\right)\left({ }^{i} H\right)$. The values $\check{a}_{r}$ and $\check{\tau}$ are affected by a STQC structure via ${ }^{0} \check{H}(70)$ with a memory ${ }^{0} \bar{\eta}$ but the parameter ${ }^{i} H$ and angular velocity for oscillations are not subjected to direct modifications.

Let us explain how the Universe is reheated both by the nonholonomic and STQC structure and oscillations during such a phase. We can use the formulas (490)-(492) in [8] rewritten for "inverse hat" values and averaged square of a respective scalar curvature $\overline{\hat{\mathbf{R}}}$, in our case, for the canonical d-connection $\widehat{\mathbf{D}}$. This redefine the system (69) for the $(\tau, \tau)$ component in the form

$$
\begin{aligned}
\check{H}^{2}+\frac{\widehat{\mathbf{R}} \check{H}}{18^{i} H}\left(1-\frac{\widehat{\mathbf{R}}^{2} \check{H}}{12}\right) & =\frac{8 \pi}{3} G\left({ }^{m} \rho\right), \\
\widehat{\mathbf{R}}^{\bullet \bullet}+3 \widehat{\mathbf{R}}\left(\check{H}+2{ }^{i} H\right) & =\nu \frac{G\left({ }^{i} H\right) \omega}{8 \check{H}} \overline{\mathbf{R}}^{2} .
\end{aligned}
$$

where $G$ is the gravitational constant, $\nu$ is the number of matter fields involved in the reheating process (during the reheating era, we exclude the massless conformal fields which are not excited) and the energy density of matter is computed

$$
{ }^{m} \rho=\frac{\nu \omega}{1152 \pi} \frac{1}{\check{a}^{4}} \int_{\check{\tau}_{r}}^{\tau} \overline{\hat{\mathbf{R}}}^{2} \check{a}^{4} d \tau \simeq \frac{3}{5} \frac{32}{1152 \pi} \frac{\nu \omega^{3}}{\tau-\check{\tau}_{r}} ;
$$

for cosmic times $\tau \gg \check{\tau}_{r}+\omega^{-1}$, the matter energy ${ }^{m} \rho$ tends to zero and we obtain radiation dominating solutions. The effective ${ }^{m} \rho$ is slightly affected by STQC structure via $\check{\tau}_{r}$ but this dependence is lost for cosmic times. In result, we can estimate the corresponding reheating and Friedmann temperatures as in $R^{2}$ gravity with standard FLRW metrics,

$$
T_{r} \simeq 24 \times 10^{17} \sqrt{{ }^{i} H} l_{P l} G e v \text { and } T_{F} \sim \nu^{-3 / 4} 10^{17}\left(36{ }^{i} H l_{P l}^{2}\right)^{3 / 4} G e v,
$$

see formulas (494)-(498) in [8] for speculations on constraints on parameter ${ }^{i} H$ following form the conditions that the reheating temperature has to be large enough in order to allow the baryogenis, remain monopole free etc.

The main conclusion of this subsection is that possible off-diagonal and STQC configurations modelled with quasi FLRW metrics do not modify substantially the reheating era in MGTs and GR. We can consider hidden quasi periodic gravitational dark energy and dark matter structures which 
contribute substantially to galactic formation etc. but such configurations do not affect substantially reheating processes.

Finally, we note that using effective data $\check{H}, \check{a}, \widehat{\mathbf{R}}$ encoding off-diagonal and/or nonholonomic STQC configurations, we can elaborate on various models of inflation, for instance, in mimetic $F(R)$ theories of gravity, topological Gauss-Bonne and other type modifications of the GR, see review of such theories in Part III of [8]. MGTs result in very different cosmological scenarios of inflation with STQC structure. We omit such considerations in this work.

\section{Late STQC dynamics, dark energy and dark matter}

The $\Lambda$ CDM (LCDM) model is important in modern cosmology because it fits a series of observational data and predicted the location and existence of the baryon acoustic oscillations. In this section, we study this model for (modified) gravity with STQC structure and discuss some phenomenological aspects of dark energy era (i.e. late-time evolution) of the Universe.

\subsection{Quasi $\Lambda$ CDM epoch from STQC structures in $F(R)$ gravity}

For MGTs, this issue is addressed and reviewed in [39, 40, 8]. It is also studied in the context of off-diagonal cosmological solutions and quasiperiodic structures in [1, 2, 3, 4, 5, 6]. We show how that approach with reconstruction techniques can be generalized for STQC configurations with effective data $\check{H}(\tau), \check{a}(\tau), \widehat{\mathbf{R}}(\tau)$, when the computations are performed for a flat quasi FLRW metric (57) when $\bar{\eta}_{i}(\tau) \simeq 1+\varepsilon \check{\chi}_{i}(\tau)$ as in (36). The effective $\Lambda$ CDM Hubble rate is $\check{H}^{2}-H_{0}^{2}=\frac{2}{3} M_{P}^{2} \rho_{0} \check{a}^{-3}$, where $\check{H}$ and $\check{a}$ encode possible STQC structure and $\rho_{0}$ and $H_{0}$ are constants, when $\check{\Lambda}=12 H_{0}^{2}$ can be identified with $\check{\Lambda}={ }^{m} \Lambda+\bar{\Lambda}$ for nonlinear symmetries (43). We can consider $\rho_{0}$ as an integration constant for all (effective) matter.

Prescribing any value of $\widehat{\mathbf{R}}[\bar{\eta}]$ encoding various possible nonlinear contributions via gravitational polarization $\bar{\eta}$ (59), we can apply the reconstruction technique [39], see also section IV-B in [8] and [2], we can encode STQC contributions into an effective $F(R)$ theory by introducing a functional

$$
G(\check{N})=\check{H}^{2}(\check{N})=H_{0}^{2}+\frac{2}{3} M_{P}^{2} \rho_{0} a_{0}^{-3} e^{-3 \check{N}}
$$

where $a_{0}$ is also an integration constant and $\check{N}$ is an e-folding function of type (71) expressed as a function of $\widehat{\mathbf{R}}, \check{N}=-\frac{a_{0}^{3}}{6 M_{p}^{2} \rho_{0}}\left(\widehat{\mathbf{R}}-12 H_{0}^{2}\right)$. This formula can be used for elaborating an analogous model with nonholonomic nonminimal coupling as in [2] when the modified Einstein equations (65) are written

$$
6\left(\widehat{\mathbf{R}}-9 H_{0}^{2}\right)\left(\widehat{\mathbf{R}}-12 H_{0}^{2}\right) F^{\prime \prime}(\widehat{\mathbf{R}})-\left(\widehat{\mathbf{R}}-18 H_{0}^{2}\right) F^{\prime}(\widehat{\mathbf{R}})-F(\widehat{\mathbf{R}})=0
$$

This equation can be solved analytically in terms of the Gauss hypergeometric function $F(\widehat{\mathbf{R}})=$ $F(\widehat{\alpha}, \widehat{\beta}, \widehat{\gamma}, \widehat{r})$ when the parameters are subjected to the conditions $\widehat{\alpha}+\widehat{\beta}=\widehat{\alpha} \widehat{\beta}=\widehat{\gamma}=-1 / 6$ and $\widehat{r}=-3+\widehat{\mathbf{R}} / 3 H_{0}^{2}$.

Finally, we note that prescribing STQC configurations we can reproduce various models of effective $F(\widehat{\mathbf{R}})$ gravity explaining a series of observational data for $F(\widehat{\mathbf{R}}) \sim R^{2}$. Following Starobinsky work [36], such theories can be modelled equivalently via interactions with a scalar field. In this work, we show that certain classes of solutions can be parameterized to describe quasiperiodic configurations. 


\subsection{STQC unification of inflation with dark energy era in MGTs}

In this section we speculate on unified description of the inflationary era with the dark energy, DE, era in the context of $F(R)$ gravity. For LC-configurations, such results are reviewed in [8]. In this sections, we study certain conditions for elaborating cosmological theories with nontrivial STQC structure. In the late-time era with effective STQC structure, we take

$$
\check{F}(\widehat{\mathbf{R}})=\widehat{\mathbf{R}}-2 \check{\Lambda}\left(1-e^{-\widehat{\mathbf{R}} / R_{0}}\right)+{ }^{i} \Lambda\left(1-e^{-\left(\widehat{\mathbf{R}} /{ }^{i} R\right)^{\varsigma}}\right)+\check{\gamma} \widehat{\mathbf{R}}^{\check{\alpha}} .
$$

In this formula, $\check{\Lambda}={ }^{m} \Lambda+\bar{\Lambda}$ and certain constants $R_{0},{ }^{i} \Lambda,{ }^{i} R, \check{\gamma}, \check{\alpha}$ will be determined from the conditions that STQC configurations are modelled equivalently both in GR and a suitable MGT and resulting in observational data. This way, the quasiperiodic structure can preserved in the limit $\widehat{\mathbf{R}} \gg R_{0}$ and realized by nonholonomic deformations or LC-configurations of the Einstein-Hilbert action and satisfying stability of the late-time de Sitter vacuum and avoiding antigravity. For such an approximation, the local corrections to the Newton law are negligible.

The essential features of the STQC $F(R)$-model can be studied using the (effective) DE evolution $\check{\rho}_{D E}=\check{\rho}_{\text {eff }}-\rho / \check{F}^{\prime}(\widehat{\mathbf{R}})$, where $\rho$ is for the standard matter fields and the quasi FLRW equation is written

$$
\check{\rho}_{\text {eff }}=\frac{3}{2 M_{P}^{2}} \check{H}^{2}=\left[\rho+\left(2 M_{P}\right)^{-2}\left(\check{F}^{\prime} \widehat{\mathbf{R}}-\check{F}-6 \check{H}\left(\check{F}^{\prime}\right)^{\bullet}\right)\right] / \check{F}^{\prime} .
$$

The conservation laws for DE are considered in a standard effective fluid form,

$$
\frac{d \check{\rho}_{D E}}{d(\ln \check{a})}+3\left(\check{\rho}_{D E}+\check{p}_{D E}\right)
$$

for a EoS parameter $\check{w}_{D E}=\check{p}_{D E} / \check{\rho}_{D E}$.

To analyze properties of the STQC F-modified Friedmann equation (65) is convenient to introduce a new variable and redefine the parameters,

$$
\check{y}:=\check{\rho}_{D E} / \rho_{m}^{(0)}=\check{H}^{2} / \tilde{m}^{2}-\check{a}^{-3}-\chi \check{a}^{-4},
$$

where the mass scale $\tilde{m}^{2}=2 M_{p}^{2} \rho_{m}^{(0)} / 3 \simeq 1.5 \times 10^{-67} \mathrm{eV}^{2}$ is computed for the energy density of matter at present time, $\rho_{m}^{(0)}$, and $\chi=\rho_{r}^{(0)} / \rho_{m}^{(0)} \simeq 3.1 \times 10^{-4}$ is determined by the energy density of radiation at present time. Approximating $\widehat{\mathbf{R}}=12 \check{H}^{2}+6 \check{H}^{\bullet}$ for $\check{H}=\ln |\check{a}|$, we express (75) for a functional $\check{y}(\check{H})$ in the form

$$
\frac{d^{2} \check{y}}{(d \check{H})^{2}}+\check{J}_{1} \frac{d \check{y}}{d \check{H}}+\check{J}_{2} \check{y}+\check{J}_{3}=0 .
$$

In this equation, there are considered functions

$$
\begin{aligned}
& \check{J}_{1}=4+\frac{1-\check{F}^{\prime}(\widehat{\mathbf{R}})}{6 \tilde{m}^{2} \check{F}^{\prime \prime}(\widehat{\mathbf{R}})\left(\check{y}+\check{a}^{-3}+\chi \check{a}^{-4}\right)}, \check{J}_{2}=\frac{2-\check{F}^{\prime}(\widehat{\mathbf{R}})}{3 \tilde{m}^{2} \check{F}^{\prime \prime}(\widehat{\mathbf{R}})\left(\check{y}+\check{a}^{-3}+\chi \check{a}^{-4}\right)}, \\
& \check{J}_{3}=-3 \check{a}^{-3}-\frac{1-\check{F}^{\prime}(\widehat{\mathbf{R}})}{6 \tilde{m}^{2} \check{F}^{\prime \prime}(\widehat{\mathbf{R}})}+\frac{\check{F}(\widehat{\mathbf{R}})-\widehat{\mathbf{R}}}{18 \tilde{m}^{4} \check{F}^{\prime \prime}(\widehat{\mathbf{R}})\left(\check{y}+\check{a}^{-3}+\chi \check{a}^{-4}\right)},
\end{aligned}
$$

when the scalar curvature and effective EoS parameter are determined by STQC structure encoded in $\check{y}$ as follows,

$$
\widehat{\mathbf{R}}=3 \tilde{m}^{2}\left(\frac{d \check{y}}{d \check{H}}+4 \check{y}+\check{a}^{-3}\right) \text { and } \check{w}_{D E}=-1-\frac{1}{3} \frac{d \ln |\check{y}|}{d \check{H}} \text {. }
$$


The constructions in this section are very similar to those in sections IV.A and IV.C of [8]. In our approach, the difference is that our variables $\check{y}, \check{a}, \check{H}$ etc. are stated by a STQC structure with effective $\check{\Lambda}={ }^{m} \Lambda+\bar{\Lambda}$. It is also convenient to work with a different system of notations in (74). We can use those numeric solutions for a range $R_{0} \ll \widehat{\mathbf{R}} \ll{ }^{i} R$, with $R_{0} / \check{\Lambda}=0.6,0.8$ and 1 , including the matter domination era and the current acceleration epoch

$$
\check{\Lambda}={ }^{m} \Lambda+\bar{\Lambda}=7.93 \tilde{m}^{2}, \quad{ }^{i} R / 2={ }^{i} \Lambda=10^{100} \check{\Lambda}, \varsigma=4, \check{\alpha}=5 / 2, \check{\gamma}=\left(4{ }^{i} \Lambda\right)^{1-\check{\alpha}} .
$$

Here we note that such results can be reproduced by choosing the initial conditions in a form similar to that for the standard $\Lambda \mathrm{CDM}$ model for a red shift $\check{z}=\check{a}^{-1}-1$. There are taken such initial conditions for $\check{z}={ }^{i} \check{z}$ when $(d \check{y} / d \check{z})_{\mid}{ }_{\check{z}}=0$ and $\check{y}_{\mid} i_{\check{z}}=\left(3 \tilde{m}^{2}\right)^{-1} \check{\Lambda}$. There is a compatibility with WMAP results if the values $i \check{z}=1.5,2.2,2.5$ are chosen respectively for $R_{0} / \check{\Lambda}=0.6,0.8$ and 1 and $\widehat{\mathbf{R}}=3 \tilde{m}^{2}(\check{z}+1)^{3}$. The respective value of the EoS parameter $\check{z}=0$ (present epoch) result respectively in values: $-\check{w}_{D E}=0.994,0.975,0.950$, which for the $\mathrm{DE}$ is very closed to the phantom line -1 . Such results prove that even the STQC structure may substantially change the vacuum and nonvacuum cosmological evolution via nontrivial gravitational polarization $\bar{\eta}$ (59) we can chose such effective variables and parameters when the models are equivalent to certain $F(R)$ theories or solutions in describing GR with off-diagonal nonlinear interactions.

\section{Conclusions}

With this article we aimed to elaborate on a new class of inflation and late time cosmological models with time quasiperiodic structures in MGTs and GR. We tried to make the paper selfconsistent, so we reviewed in brief our geometric method on constructing generic off-diagonal locally anisotropic cosmological solutions and analyzed possible nonholonomic constraints and parametric decompositions resulting in diagonal and effective $\Lambda$ CDM models encoding space-time quasiperiodic, STQC, structure. Following results of Planck2015 [37, 38] (that the ration of tensor perturbation amplitude $r<0.1$ ), a number of researchers concluded that such observational data seem to "virtually eliminate all the simplest textbook inflationary models". In order to solve this problem and update cosmological scenarios, theorists elaborate on MGTs and constructing new classes of exact and parametric solutions. Former methods of transforming generalized Einstein equations into systems of nonlinear ordinary differential equations with solutions determined by integration constants fail in elaborating accelerating cosmology scenarios with quasiperiodic structure and locally anisotropic and inhomogeneous evolution.

It is well-known that modern cosmological data (see details and discussions in [12, 8, 17, 16, 1, 39, 40, 37, 38]) prove that the acceleration of the Universe is determined by a new dark energy, DE, and dark matter, DE, physics. This resulting in existence of complex network filament, quasiperiodic and aperiodic like structures (with various nonlinear wave interactions, diffusion processes, fractal like configurations etc.). Such "hidden" and directly observed rich geometric gravitational field/ spacetime and (effective) matte structures are driven by a nonlinear cosmological evolution with respective scale dependence for meta-galactic and galactic configurations. It is considered that such modified cosmological models can be elaborated realistically only for a new class of MGTs even for certain cases the GR seem to be correct if, for instance, nonlinear locally anisotropic and inhomogeneous cosmological solutions with generic off-diagonal terms for metrics are considered. The formation and evolution of cosmological configurations with nonlinear gravitational and matter field 
structures can be modelled by numeric, analytic and geometric methods as deformations of an effective quasycristal, QC, space structure for the gravitational and fundamental scalar fields, see [3, 5, 6] and references therein. It should be emphasized that we can not describe quasiperiodic and pattern forming structures as exact solutions of (modified) Einstein equations if we work with cosmological models based only on the Friedman-Lamaitre-Robertson-Worker, FLRW, metric and/or standard Bianchi anisotropic generalizations with metrics depending only a time like variable and strcutre Lie group symmetries. Using such "simplified" ansatz for metrics, we can transform the gravitational and matter field equations into certain systems of nonlinear ordinary differential equations, ODEs, and certain algebraic matrix equations. Such equations can be solved in terms of integration constants but this does not allow, for instance, to obtain nonlinear/ solitonic waves (or other type cosmological complex structures like QC, networks and filaments) which involve generic off-diagonal solutions on nonlinear partial differential equations, PDEs, expressed in terms of generation and integration functions, parametric nonlinear dependencies etc. We have to elaborate more advanced geometric methods in order to solve analytically such physically important systems of PDEs. Using only numeric and approximate methods result in "non-exact" solutions which do not provide a complete understanding of properties of corresponding nonlinear dynamics.

Our anholonomic frame deformation method, AFDM, [1, 2, 3, 6] allows to construct more realistic classes of exact and parametric solutions determined by generating and integration functions and (effective) matter sources containing, in general, possible modifications of GR from classical and quantum gravity theories. The advantage of the AFDM is that it allows us to chose generating functions and sources in various forms describing STQC-, QC-, pattern forming, nonlinear wave etc. structures as exact and parametric cosmological solutions of generalized Einstein equations both in MGTs and GR. In general, such solutions are inhomogeneous and locally anisotropic and described by generic off-diagonal metrics, non-Riemann connections, with coefficients depending, in principle, on all spacetime coordinates and subjected to certain nonholonomic constraints and generalized symmetries. Nevertheless, it is always possible to impose at the end some additional constraints (for instance, the condition of zero torsion, certain observable boundary behaviour and symmetries, or to consider homogeneous and isotropic configuration with nontrivial topology) and search for possible limits to known cosmological scenarios. We emphasize here that if we impose certain fixed high symmetry conditions and chose a "very simplified" ansatz from the very beginning (before finding generalized solutions for nonlinear systems of PDEs), the possibility to construct general self-consistent cosmological scenarios with quasiperiodic and pattern forming structures is lost. Such configurations can not be completely described in terms of ODEs and their particular solutions. We have to involve more general classes of solutions of (modified) Einstein equations transformed into corresponding effective PDEs in order to explain and predict modern cosmology observational and experimental data.

The novelty of this work is that we developed the AFDM in a form which allows to generate cosmological STQC-type structures both for the gravitational and matter fields (which is important for elaborating new theories of DE and DM physics) as exact and parametric solutions. Our new classes of cosmological solutions prove that nonlinear gravitational and matter field interactions induce quasiperiodic structures which are generalizations (in certain approximations, similar) of time crystals constructed as analogous to space crystals [19] but those models where elaborated for generalized nonlinear mechanics, classical and quantum dynamical systems and condensed matter physic and not for gravity theories and cosmology. For quasiperiodic space configurations, such structures model respective spacetime QC structures as we elaborated in our previous works [3, 5, 6]. 
The primary emphasis of quantum theory in formatting classical time crystals in solid state physics is analyzed in [18]. In our work (it is the first one on STQC), we study how analogous configurations can generated as (off-) diagonal cosmological configurations in MGTs and GR. In particular, it is shown that various (quasi) periodic gravitational and matter field structures can be generated by a spontaneous broken gravitational vacuum with different nonlinear symmetries. We can motivate that the early universe can be described by a STQC gravitational dynamics and filled, for instance, with DM time quaisperiodic structures by considering a more complete nonlinear cosmological dynamics determined by exact solutions of generalized Einstein equations. Such exact solutions can be redefined in arbitrary systems of reference/coordinates. So, prescribing gravitational/matter field generating functions with time-dependence and quasiperiodicity (for instance, in a system of reference where the system of field equations were decoupled in a general form) we can recompute such values for any choice of a time coordinate in a physical system of reference. This is possible because the AFDM method allows to integrate physically important systems of PDEs in general exact or parametric forms and to consider such solutions in any geometric/ physical system of reference. If we would work with approximate numeric methods, the type of time dependence and quasiperiodicity conditions would mix the coefficients of metrics and connections and affect substantially the possibility of decoupling and integrating explicitly such nonlinear equations. This is another important priority of the AFDM, see [1, 2, 3, 4, 5, 6] and references therein. Even there were published many papers on cosmological impacts of $f(R)$ - and $f(T)$-theories, the dynamics of the universe etc. described by other classes of less general solutions, those constructions could not prove the existence and encode STQC-structures. In sections 4 and 5, we elaborated respectively on such time quasiperiodic inflationary and acceleration cosmology models and proved that such STQC-models describe more realistically modern observational data.

In condensed matter physics, the subject of dynamical fluctuations of time crystals was investigated in [41, 42]. We refer to those works for detailed explanations on how effective field theories can be derived. In our case, we study the self-consistent small parametric evolution of quantum fluctuations which can be encoded as locally anisotropic cosmological structures modelling space-time crystals. Such dynamical systems posses a local conventional lowest energy and performs a periodic motion. STQC cosmological dynamics involve a more rich nonlinear pattern forming structure, with memory of nonlinear effects. This can be used for more realistic elaboration of dark energy and dark matter models.

Finally, we note that our approach is based on the hypothesis that inflation is primarily driven by vacuum energy at a scale indicated by gauge coupling unification [43. Concretely, in this paper, we elaborated a class of models with hybrid inflations for which the vacuum energy is associated with a grand unified theory condensate. Such a quasi-periodic analogous condensate provides the dominant energy during inflation and a second inflation scalar slow-rolls. In a more general context, we can consider quantum corrections and modified dispersion relations which is a goal for our future research.

Acknowledgments: This work consists a natural extension of the research program for the project IDEI in Romania, PN-II-ID-PCE-2011-3-0256. Author thanks D. Singleton for hosting his adjunct position at Fresno State University. He is also grateful for references on time crystals (related to A. Shapere and F. Wilczek works [18, 19]) and important discussions on former works on locally anisotropic quasiperiodic structures in (modified) gravity and cosmology [1, 2, 3, 6]. 


\section{References}

[1] S. Vacaru, Equivalent off-diagonal cosmological models and ekpyrotic scenarios in $f(R)$-modified massive and Einstein gravity, Eur. Phys. J. C 75 (2015) 176; arXiv: 1504.04346

[2] E. Elizalde and S. Vacaru, Effective Einstein cosmological spaces for non-minimal modified gravity, Gen. Relat. Grav. 47 (2015) 64; arXiv: 1310.6868

[3] S. Vacaru, Off-diagonal ekpyrotic scenarios and equivalence of modified, massive and/or Einstein gravity, Phys. Lett. B 752 (2016) 27-33; arXiv: 1304.1080S.

[4] S. Rajpoot and S. Vacaru, Cosmological Attractors and Anisotropies in two Measure Theories, Effective EYMH systems, and Off-diagonal Inflation Models, Eur. Phys. J. C 77 (2017) 313; arXiv: 1610.01090

[5] M. M. Amaral, R. Aschheim, L. Bubuianu, K. Irwing, S. Vacaru, and D. Woolridge, Anamorphic quasiperiodic universes in modified and Einstein gravity with loop quantum gravity corrections, Class. Quant. Grav. 34 (2017) 185002; arXiv: 1611.05295

[6] L. Bubuianu and S. Vacaru, Deforming black hole and cosmological solutions by quasiperiodic and/or pattern forming structures in modified and Einstein gravity, Eur. Phys. J. C 78 (2018) 393; arXiv: $1706.02584 \mathrm{v} 3$

[7] S. Capozziello and V. Faraoni, Beyond Einstein Gravity (Springer, Berlin, 2010)

[8] S. Nojiri and S. Odintsov, Unified cosmic history in modified gravity: from $F(R)$ theory to Lorentz non-invariant models, Phys. Rept. 505 (2011) 59-144; arXiv: 1011.0544

[9] D. S. Gorbunov and V. Rubakov, Introduction to the theory of the early universe: Cosmological perturbations and inflationary theory (Hackensack, USA: World Scientific, 2011)

[10] T. Clifton, P. G. Ferreira, A. Padilla and C. Skordis, Modified gravity and cosmology, Phys. Repts. 512 (2012) 1-189; arXiv: 1106.2476

[11] S. Basilakos, A. Pl Kouretsis, E. N. Saridakis and P. Stavrinos, Resembling dark energy and modified gravity with Finsler-Randers cosmology, Phys. Rev. D 88 (2013) 123510, arXiv: 1311.5915

[12] A. Linde, Inflationary cosmology after Plank 2013, in: Post-Planck Cosmology: Lecture Notes of the Les Houches Summer School: Vol. 100, July 2013: editors C. Deffayet, P. Peter, B. Wandelt, M. Zadarraga and L. F. Cugliandolo (Oxford Scholarship Online, 2015); arXiv: 1402.0526

[13] R. Gwyn, G. A. Palma, M. Sakellariadou and S. Sypsas, On degenerate models of cosmoc inflation, JCAP 1410 (2014) 005; arXiv: 1406.1947

[14] M. Wali Hossain, R. Myrzakulov, M. Sami and E. N. Saridakis, Unification of inflation and dark energy á la quintessential inflation, Int. J. Mod. Phys. D 24 (2015) 1530014

[15] S. Pabilakos, N. E. Mavromatos and J. Sola, Starobinsky-like inflation and running vacuum in the context of supergravity, Universe 2 (2016) 14, arXiv: 1505.04434 
[16] S. Nojiri, S. D. Odintsov and V. K. Oikonomou, Modified gravity theories on a nutshell: inflation, bounce and late-time evolution, Phys. Rep. 692 (2017) 1-104; arXiv: 1705.11098

[17] S. Vagnozzi, Recovering a MOND-like acceleration law in mimetic gravity, Class. Quan. Grav. 34 (2017) 185006; arXiv: 1708.0063

[18] A. Shapere and F. Wilczek, Classical time crystals, Phys. Rev. Lett. 109 (2012) 160402; arXiv: 1202.2537

[19] F. Wilczek, Quantum time crystals, Phys. Rev. Lett. 109 (2012) 160401; arXiv: 1202.2539

[20] F. Wilczek, Wilczek reply: Phys. Rev. Lett. 110 (2013) 118902

[21] F. Wilczek, Superfluidity and space-time translation symmetry breaking, Phys. Rev. Lett. 111 (2013) 250402; arXiv: 1308.5949

[22] A. D. Shapere and F. Wilczek, Realization of "time crystal" Lagrangians and emergent sisyphus dynamics, arXiv: 1708.3348

[23] K. Sacha and J. Zakrzewski, Time crystals: a review, Rep. Prog. Phys. 81 (2018) 016401; arXiv: 1704.03735

[24] T. Li, Z.-X. Gong, Z.-Q. Yin, H. T. Quan et all., Space-time crystals of trapped ions, Phys. Rev. Lett. 109 (2012), arXiv: 1206.4772

[25] N. Y. Yao, A. C. Potter, I. -D. Potirniche, and A. Vishwanath, Dicrete time crystals: rigidity, criticality, and ralizations, Phys. Rev. Lett. 118 (2017); arXiv: 1608.02589

[26] S. Choi, J. Choi, R. Landig et all., Observation of discrete time-crystalline order in a disordered dipolar many-body system, Nature 543 (2017) 221-225; arXiv: 1610.08057

[27] J. Zhang, P. W. Hess, A. Kyprianidis et all., Observation of a discrete time crystal, Nature 543 (2017) 217-220; arXiv: 1609.08684

[28] P. Bruno, Comment on "Quantum time crystals", Phys. Rev. Lett. 110 (2013) 118901; arXiv: 1210.4128

[29] P. Bruno, Comment on "Space-time crystals of trapped ions", Phys. Rev. Lett. 111 (2013) 029301; arXiv: 1211.4792

[30] P. Nozières, Time crystals: Can diamagnetic currents drive a charge density wave intor rotation? EPL (Europhysics Letters) 103 (2013) 57008; arXiv: 1306.6229

[31] G. E. Volovik, On the broken time translation symmetry in macroscopic systems: Processing states and off-diagonal long-range order, JETP Letters 98 (2013) 491-495; arXiv: 1309.1845

[32] H. Watanabe and M. Oshikawa, Absence of quantum time crystals, Phys. Rev. Lett. 114 (2015) 251613; arXiv: 1410.2143 
[33] R. Yoshii, S. Takada, S. Tsuchiya, G. Marmorini, H. Hayakawa, and M. Nitta, Fulde-ferrellLarking-Ovchinnikov states in a superconducting ring with magnetic fields: Phase diagrams and the first-order phase transitions, Phys. Rev. B 92 (2015) 2245128; arXiv: 1404.3519

[34] D. V. Else, B. Bauer, C. Nayak, Floquet time crystals, Phys. Rev. Lett. 117 (2016) 090402; arXiv: 1603.08001

[35] V. Khemani, A. Lazaridees, R. Moessner, and S. L. Sondhi, Phase structures of driven quantum systems, Phys. Rev. Lett. 116 (2016) 250401; arXiv: 1508.03344

[36] A. A. Starobinsky, A new type of isotropic cosmological models without singularity, Phys. Lett. B 91 (1980) 99

[37] Planck 2015 results. XIII. Cosmological parameteres, v3, 17 Jun 2016, arXiv: 1502.01589

[38] Planck 2015 results. XX. Constraints on inflation. v1, 7 Feb 2015, arXiv: 1502.02114

[39] S. Nojiri, S. Odintsov and D. Saez-Gomez, Cosmological reconstruction of realistic modified F(R) gravities, Phys. Lett. B 681 (2009) 74, arXiv: 0908.1269

[40] P. K. Dunsby, E. Elizalde, R. Goswami, S. Odintsov and D. Saez-Gomez, On the LCDM Universe in $\mathrm{f}(\mathrm{R})$ gravity, Phys. Rev. D 82 (2010) 023519, arXiv: 1005.2205

[41] E. Castillo, B. Koch, and G. Palma, On the integration of fields and quanta in time dependent backgrounds, JHEP 05 (2014) 111; arXiv: 1312.3338

[42] E. Castillo, B. Koch, and G. Palma, On the dynamics of fluctuations in time crystals, arXiv: 1410.2261

[43] M. P. Hertzberg and F. Wilczek, Inflation driven by unification energy, Phys. Rev. D 95 (2017) 063516; arXiv: 1407.6010 